\title{
Fundamental Aspects of Dislocation Theory A Conference Report with Abstracts
}

\author{
John A. Simmons and Roland deWit \\ Metallurgy Division, Institute for Materials Research
National Bureau of Standards, Washington, D.C. 20234
and
}

R. Bullough

Theoretical Physics Division, A.E.R.E. Harwell, Didcot, Berkshire, England

\section{Conference Reporf}

The conference on "Fundamental Aspects of Dislocation Theory" was held under the auspices of the Institute for Materials Research at the Gaithersburg Laboratories of the National Bureau of Standards on April 21-25, 1969.

The purpose of the Conference was to bring together workers active in all fundamental aspects of the theory of dislocations to discuss, evaluate, and contribute to the current state of understanding of these defects in crystalline materials.

The dislocation has been a subject of theoretical study for some 60 years, starting with the investigations reported by Volterra. In the last 20 years there has been a steady increase in sophistication of dislocation theory from the early almost intuitive models to current theories, which range over a large portion of continuum mechanics and solid state physics-from the nonRiemannian geometry of the continuum to anharmonic calculations of atomic configurations and phonon scattering.

A sustained interest in dislocations has existed on account of their anticipated applications, particularly to mechanical properties of materials, but the study of dislocations has received an added impetus over the last 15 years by better correlations with experimental observations made possible by advances in such fields as electron microscopy and crystal growth. With this increase in experimental work recent conferences have been predominantly devoted to experimental results. We felt, therefore, that this was a suitable time for a conference whose scope was limited enough to allow some in-depth treatment of various theoretical developments in the study of dislocations.

A small closed conference of about sixty people was decided upon to insure a high degree of informality and interaction. However, towards the end of our preparations the general interest had exceeded our expectations, so that a little over one hundred participants with forty percent drawn from eighteen different foreign countries finally took part; this produced a gathering of truly international character.

The conference was organized into ten sessions with over seventy contributed papers; the abstracts of the papers are listed alphabetically following this report below. Each chairman organized the papers in his session according to his own views. Two panels punctuated the sessions; these provided some perspective on current opinion of dislocation theory and its relevance to practical problems by aiming at such questions as: "How far have we come?" and "Where do we go from here?" The explicit agenda was as follows:

1) Discrete Dislocations in Continuum Elasticity Chairmen: J. D. Eshelby and R. deWit

2) Lattice Theories Chairman: R. Bullough

3) Dislocation-Phonon Interactions Chairman: J. Lothe

4) Applications of the Geometry of Dislocations Chairman: F. R. N. Nabarro

5) Panel on Intrinsic Properties of Dislocations Chairman: J. P. Hirth

6) Field Theories, Part I Chairman: E. Kröner

7) Field Theories, Part II Chairman: J. A. Simmons

8) Thermally Activated Processes and Statistical Theories

Chairman: A. Seeger

9) Dislocation-Electron Interactions Chairman: R. Thomson

10) Panel on Future Directions for Dislocation Theory

Chairman: A. Seeger 


\section{Session Papers and Summaries}

\subsection{Discrete Dislocations in Continuum Elasticity}

A. K. Head: Discrete Dislocations in Continuum Elasticity

J. Lothe: The Image Force on Dislocations at Free Surfaces. Comments on the Concept of Line Tension

K. Malén: Stability and Some Characteristics of Uniformly Moving Dislocations

G. P. Senkeckyj: Screw Dislocations in Inhomogeneous Solids

D. J. Bacon and P. P. Groves: The Dislocation in a Semi-Infinite Isotropic Medium

D. Kuhlmann-Wilsdorf and R. F. Duncan: Theoretical Considerations on the Extension of $1 / 2\langle 110\rangle$ $\{111\}$ Dislocations in Isotropic FCC Metals into Shockley Partials

J. Weertman, H. *M. Berg, J. E. Bloom, H. Ishii, R. H. Marion, D. E. Pease, D. T. Spreng and J. B. Vander Sande: Subsonic, Supersonic, and Transonic Dislocations Moving on an Interface Separating Two Media of Differing Elastic Properties

J. A. Simmons and R. Bullough: Internal Stress and the Incompatibility Problem in Infinite Anisotropic Elasticity

D. M. Barnett: Series Representations of the Elastic Green's Tensor for Cubic Media

N. Louat: Some Problems Involving Linear Dislocation Arrays

J. C. M. Li: Some Recent Results on Dislocation Pileups

E. Smith: The Behavior of an Elastic Solid Containing Distributions of Free and Fixed Dislocations

M. O. Tucker: The Elastic Interaction Between Grain Boundaries and Screw Dislocation Pile-ups

The session dealt with current research in what may be called "classical dislocation theory," i.e. the theory formulated about 15 years ago in terms of linear elasticity. Two main points stood out: The concept of the force on a straight but finite dislocation segment, and the advantage of using the elastic Green's tensor. The force concept was used by one conferee to derive a surprisingly simple expression for the image force on a dislocation emerging at a free surface, and by another to find a stability criterion for a moving dislocation in an anisotropic medium. The power of the Green's tensor in three dimensions for anisotropic bodies was shown in the context of the general internal stress problem, and a related decomposition theorem (somewhat analogous to Helmholtz' theorem) was presented using projection operators. Then a convenient series representation in terms of the anisotropy factor of the Green's tensor for cubic media was given. This session was so crowded that several interesting papers could not be presented orally, but only discussed from the floor.

\subsection{Lattice Theories}

N. W. Ashcroft: One Electron Theories of Cohesion on Ion-Pair Potentials in Metals

A. A. Maradudin: Localized Vibration Modes Associated with Screw Dislocations

J. W. Flocken and J. R. Hardy: The Method of Lattice Statics

H. Suzuki: Effect of Zero-Point Motion on Peierls Stress

A. Englert, H. Tompa and R. Bullough: Point Defects and Dislocations in Copper

M. Doyama and R. M. J. Cotterill: Atomistic Calculation of Dislocation in Solid Krypton

A. Hölzler and R. Siems: A Lattice Theory Model for Peierls-Energy Calculations

R. Chang: The Interaction Between a Screw Dislocation and Carbon in Body-Centered Cubic Iron According to an Atomic Model

P. C. Gehlen, G. T. Hahn and A. R. Rosenfeld: The Structure of the $\langle 111\rangle$ Screw Dislocation in Iron

Many of the mechanical and physical properties of dislocations can only be fully understood when we have knowledge of their core structure and energy. To obtain such information demands a priori knowledge of the cohesive energy of the crystal lattice, particularly when the atom configuration is far from that of the perfect crystal. In addition the complex topology of the dislocation means that reasonable calculations are only possible if the cohesive energy can be represented in a simple spatial form, such as an analytic pair potential or a simple force constant model.

The session was opened with a survey of the electron theories of cohesion in metals which was a critique of the physical validity of the use of ion-pair potentials in metals. The force constant models were then discussed both in the dynamic situation of phonon scattering (flutter) and in the static situation for the harmonic static configurations - although the latter work was confined to more elementary point defects. Subsequent discussion suggested that the early calculations of 
Maradudin on the simple screw dislocation could now be usefully extended to a lattice statics study of the edge dislocation. The problems involved in calculating the Peierls stress and the effects of zero point energy were discussed in relation to the various pair potential models and the session was concluded by a series of research contributions on dislocations in copper, krypton and iron. It was clear that with the rapid developments in computer speed and capacity very ambitious lattice calculations are possible; however it was felt that emphasis should be placed upon the construction of physically valid interatomic potentials and not upon ambitious relaxation procedures.

\subsection{Dislocation - Phonon Interactions}

T. Ninomiya: Eigenfrequencies in a Dislocated Crystal P. Gruner: Phonon Scattering by Dislocations and its Influence on the Lattice Thermal Conductivity and on the Dislocation Mobility at Low Temperatures

P. G. Klemens: Phonon Scattering by Cottrell Atmospheres

A. Seeger and H. Engelke: Dragging Forces on Moving Defects by Strain Field Phonon Scattering

J. H. Weiner: Thermal Energy Trapping by Moving Dislocations

J. A. Garber and A. V. Granato: Dislocation Resonance R. O. Schwenker and A. V. Granato: Dislocation Radiation

C. Elbaum and A. Hikata: The Anharmonic Properties of Vibrating Dislocations

W. P. Mason: A Source of Dissipation that Produces an Internal Friction Independent of the Frequency

This busy session dealt not only with conventional dislocation-phonon interactions but also took up the topic of dislocation drag. It was divided into four subsections, the first two of which treated conventional phonon scattering theories of fluttering and anharmonic scattering.

Dislocation fluttering, which occurs even in a harmonic theory, is produced by the effective inertia of a dislocation which causes impinging phonons to be scattered with a subsequent fluttering of the dislocation line. A review of the perturbations of the eigenfrequencies of a dislocated continuum was presented in this subsession and was related to fluttering for special dislocation configurations.

The second subsession, which was devoted to anharmonic scattering, contained two contributions. A review of scattering in the continuum was given with non-linear elastic effects taken into account. Par- ticular attention was paid to the influence of dislocation configurations and boundary effects on the phonon contribution to thermal conductivity. The second presentation was concerned with the anharmonic effects of Cottrell atmospheres on thermal conductivity particularly after plastic deformation when thermal resistivity can be expected to change as the atmospheres reform by diffusion.

The third subsession turned to the topic of dissipative drag on moving dislocations. Under this title are included such mechanisms as drag by kinks, impurities, or Peierls forces and accoustic radiation from rapidly moving dislocations. Though these topics are not strictly dislocation-phonon interaction processes, the similarity of techniques made the contributions particularly appropriate here. This subsession essentially extended into the fourth subsession which was devoted primarily to the different vibrating string models of dislocations, their experimental applications, and their correlations with dislocation drag.

\subsection{Applications of the Geometry of Dislocations}

W. Bollman: The Meaning of Dislocations in Crystalline Interfaces

M. H. Yoo and B. T. M. Loh: Structural and Elastic Properties of Zonal Twin Dislocations in Anisotropic Crystals

S. Mendelson: Non-Planar Dissociations of Dislocations

M. J. Marcinkowski: Propagation of Glide Through Internal Boundaries

R. Thomson: Kinks, Vacancies, and Screw Dislocations W. F. Harris: Topological Restriction on the Distribution of Defects in Surface Crystals and Possible Biophysical Applications

F. R. N. Nabarro: Disclinations in Surfaces

M. Kléman: Aspects in Liquid Crystals Relevant to the Theory of Dislocations

K. H. Anthony: Non-Metric Connexions, Quasidislocations and Quasidisclinations. A Contribution to the Theory of Non-Mechanical Stresses in Crystals

R. deWit: Linear Theory of Static Disclinations

Under this heading there appeared mainly two topics: interfaces and disclinations. The session started with a presentation of dislocations in crystalline interfaces. A generalized development of coincidence sites involving two arbitrary interpenetrating lattices was given using what amounts to the idea of modular arithmetic in each of the lattices. Generalized dislocations in interfaces were described in this context and then further extended to include the concept of secon- 
dary dislocations or "super-dislocations" with fractional Burger's vectors which occur in small atomic repeat patterns produced by lattice mismatches at interfaces; such patterns were beautifully illustrated by the use of Moiré patterns. It was then shown that such purely geometrical descriptions of interface dislocations can often be used to calculate relative boundary orientations of two phase mineral systems by employing a geometric measure which ought to vary monotonically with the grain boundary energy. It was suggested in subsequent discussion that such an approach might be applied to predict martensitic habit planes for which there is currently only a strictly phenomenological theory.

Disclinations, which have been under study in isolated localities for some 10 years, were presented in their multifarious forms: in liquid crystals; on surface crystals - with possible biophysical applications - and in ordinary crystals, where their existence is subject to doubt. The discussion of these drastic defects certainly exposed their probable importance in systems of low cohesive energy.

\subsection{Intrinsic Properties of Dislocations}

Panel Members: J. P. Hirth, R. Bullough, R. deWit, C. Elbaum, J. D. Eshelby, P. Haasen, P. B. Hirsch

The agenda of this panel was as follows:

1) CORE STRUCTURES IN METALS: e.g. Peierls Forces, Atomic Calculations, Nonlinear Elasticity, Adsorption, Faults (bcc), Anisotropic Elasticity.

2) DISLOCATION DRAG MECHANISMS: e.g. Damping, Inertial Effects, Electron Interactions, Thermoelastic, Magnetic (domain wall).

3) CORE STRUCTURE IN COVALENT AND POLAR MATERIALS: e.g. Jogs, Kinks, Diffusion, Interaction with Conduction Electrons and Holes, Interaction with Ionic Defects.

4) EQUILIBRIUM CONFIGURATIONS: e.g.

Disclinations, Dipoles, Junctions, Super and Unit Jogs, Grain Boundaries and Interfaces, Nodes, Fault Tetrahedra.

5) CRITICAL CONFIGURATIONS: Cross-slip, Intersection, Bow-out, Peierls, Quasi-Peierls (bcc).

The difficult task of assessing the current status of dislocation theory was undertaken by the panel with skill and enthusiasm.
The physical significance and problems associated with dislocation core studies were reviewed and particular attention was drawn to the dangers of using inadequate ion-ion potentials. It was suggested that meaningful calculations could now be made on aluminum - these would be particularly opportune in view of the recent electron microscope observations of core configurations in this metal. The problem of ensuring complete stability for the body centered cubic latiice with its many possible fault planes was emphasized and a new "third neighbor" potential for iron was presented and discussed. In view of our rather better knowledge of the interatomic potentials for the ionic solids it was suggested that more effort could usefully be put into core calculations in these materials.

The various dislocation drag processes were reviewed and it was concluded that only one-the thermo-elastic damping process - could safely be ignored. It was emphasized that the study of these drag phenomena necessitates a knowledge of both fundamental solid state physics and dislocation theory-a rare combination in one person. The only real hope appears to be for more relevant collaborative studies. The damping due to electron scattering was discussed at length and the theoretical issues distinguishing the two schools of thought on the subject were clearly delineated. Unfortunately the experimental correlation will not be completely clear until someone manages to perform low temperature ultra-sonic attenuation measurements in body centered cubic metals.

The relevance of disclinations to dislocation theory was also assessed. The consensus seemed to be that they were of much more philosophical than practical interest; their energy would be too high in a real crystal. However, in pseudocrystals, such as that made by flux lines, in liquids, two-dimensional crystals, or thin foils, they might play an important role. The evolution of the name "disclination" was revealed by Professor Nabarro: "Well, I think this is just a typical piece of bogus history, but as I remember, F. C. Frank's account of it is that he used the word "disinclination." He then wondered if this was the wisest word, and consulted the Professor of English at the University of Bristol, whose name is forgotten, and he said he was disinclined to use that word." As a companion to the "wedge" disclination, the name "twist" disclination was proposed, in analogy with the well-established names screw and edge dislocation. Discussions followed on whether it would be worthwhile to find the stacking fault energy from anisotropic calculations of the self energy of stacking fault tetrahedra and on whether lattice statics 
calculations could provide continuum theory with a better dislocation core cut-off parameter, but no satisfactory answers were given.

- At this point the allotted time had been exhausted and the last point of the agenda was skipped.

\subsection{Field Theories: Part I}

E. Kröner: The Problem on Non-Locality in the Mechanics of Solids: Review on Present Status

E. Kröner and B. K. Datta: Non-Local Theory of Elasticity for a Finite Inhomogeneous Medium - A Derivation from Lattice Theory

I. A. Kunin and A. M. Waisman: On Problems of the Non-Local Theory of Elasticity

K. Kondo: A Non-Riemannian Construction of Variational Criteria for Plastic Manifolds with Special Reference to the Theory of Yielding

M. Mișicu: Derivation of a Continuum Theory of Dislocations on the Basis of an Estimative Analysis of Crystal Lattices

R. Stojanović: The Elastic Generalized Cosserat Continuum with Incompatible Strains

D. M. Barnett: A Note on Admissible Solutions in Non-Local Elasticity

C. Teodosiu: A Dynamic Theory of Dislocations and its Applications to the Theory of the Elastic-Plastic Continuum

C. Teodosiu and A. Seeger: Non-Linear Dynamic Problems for Anisotropic Elastic Bodies in the Continuum Theory of Dislocations

C.-C. Wang: On the Thermodynamics of Inhomogeneous Bodies

C. S. Hartley and M. A. Eisenberg: Elastic-Plastic Plane Bending of a Single Crystal

S. I. Ben-Abraham: Generalized Stress and Non-Riemannian Geometry

The shortcomings of the characterization of dislocations and more complex defects in crystal lattices by means of classical linear elasticity are now accepted. Most of the papers in the first session on field theories were devoted to a re-examination of the transition from a model of an imperfect crystal lattice to a continuum theory encompassing more detailed and sophisticated defect descriptions than are contained in the classical approach.

Long range atomic interactions and lattice dispersion were discussed in the context of non-local continuum theory. In addition, the pitfalls in formulating non-local continuum theory were clearly brought out. These dif- ficulties arise from boundary effects in real finite bodies and the apparent non-existence of Green's functions in linear non-local theory when the Euler-McLauren approach is taken. A possible method for avoiding this latter difficulty by using a formulation based on truncated Fourier transforms was also presented.

The first session concluded with an attempt to combine the techniques of continuously distributed dislocation with those of engineering plasticity to a model of simple glide in a single crystal.

\subsection{Field Theories: Part II}

T. Mura: The Elastic Field of Moving Dislocations and Disclinations

S. I. Ben-Abraham: Kinematics of Continuously Distributed Dislocations

J. A. Simmons: A Geometric Foundation for the Kinematics of the Imperfect Continuum

A. C. Eringen and W. D. Claus, Jr.: A Micromorphic Approach to Dislocation Theory and its Relation to Several Existing Theories

N. Fox: On the Continuum Theory of Dislocations

The second session was opened by two survey papers on dynamical field theories of dislocations. Dynamic theories are currently in an earlier stage of development than the corresponding theories of static character. Until recently, in fact, research has been separated into general kinematic theories of a geometrical nature and linear elastic field theories and the survey papers presented were devoted to each of these two topics.

New research trends are now appearing, particularly under the impetus of continuum mechanists who are being attracted to dislocation theory. Individual papers of this type were presented in both sessions on such topics as the application of micromorphic mechanics and oriented continua to developing a thermodynamics of the deforming dislocation continuum and on the non-linear elastodynamics of dislocations. Discussion on these topics included an interesting comparison of the oriented continuum approach with the micromorphic approach. The former emphasizes separation of the incompatible lattice distortions from the motion of the points of the continuum at which they are defined, while the latter attempts to treat the continuum as a conglomeration of small elements with microstructure by introducing incompatible deformations into each of these small elements. 


\subsection{Thermally Activated Processes and Statistical Theories}

W. Frank: Dislocation Dynamics in the presence of a Multiple Spectrum of Thermally Surmountable Barriers

U. F. Kocks: Flow and the Arrhenius Equation in the Statistical Framework

A. J. E. Foreman, P. B. Hirsch and F. J. Humphreys: Movement of a Dislocation Through Random Arrays of Point and Parallel Line Obstacles

W. deRosset and A. V. Granato: Strain Rates in Dislocation Dynamics

M. S. Duesberry and P. B. Hirsch: On the Mechanism of Cross-Slip of Dislocations at Particles

H. Engelke: Theories of Thermally Activated Processes and Their Application to Dislocation Motions in Crystals

A. M. Stoneham: The Broadening of Resonance Lines by Dislocations

R. O. Scattergood and U. F. Kocks: Dislocation Pair Interaction in a Finite Body

M. Wilkens: Theoretical Aspects of Kinematical X-Ray Diffraction Profiles from Crystals Containing Dislocation Distributions

J. Holder and A. V. Granato: Thermodynamic Properties of Solids Containing Dislocations

The topics covered by this session included individually thermally activated processes involving dislocations, the statistics of thermal activation, and the statistical properties of static distributions of dislocations. The session was begun by an extensive exposition of the problem of analyzing the effective activation energy for dislocation motion in the presence of a statistical distribution of individual barriers of varying activation and back stress characteristics. Comparison was made between this theory and various other theories describing dispersion hardening, which have generally had the defect of being imprecise or essentially athermal. Following two talks on thermally activated processes, the broadening of resonance lines due to the strain arising from random distributions of static dislocations was discussed. This latter work suggests a possible new experimental technique for probing the dislocation distributions in crystals; it also may provide information on "atmosphere formation" if the resonance centers are themselves mobile and migrate to the dislocations.

\subsection{Dislocations - Electron Interactions}

P. Haasen and W. Schröter: Charged Dislocations in the Diamond Structure

C. Nourtier and G. Saada: Electronic Effects Associated with Stacking Faults in Normal Metals

R. Thomson: Theory of Surface States on Stacking Faults

C. Elbaum and Hikata: Interactions of Dislocation with Electrons in Metals

C. Elbaum and R. R. Holmes: Electronic Energy States of Dislocations; The Case of Covalent-Ionic Solids

G. P. Huffman and N. Louat: Interactions Between Electrons and Moving Dislocations

The electronic properties of dislocations in semi-conductors have received considerable theoretical attention. The various models were initially discussed and it was clear that the old dangling bond description - at the edge of the extra half plane in the core of an edge dislocation in the diamond structure-provides an inadequate model to explain the recent experimental observations on such deformed materials.

Two contributions on the topical and somewhat controversial subject of electron scattering by dislocation were presented. These treatments contrasted in both approach and results. The one approach using a Boltzmann formalism predicts the dislocation drag to be inversely proportional to the electrical resistivity and therefore to be temperature dependent-although the splitting of dislocations in fcc materials implies a small temperature dependence in that case. The alternate approach involves treating the distortion field of a moving dislocation as a phonon distribution and calculating the associated electron-phonon interactions. This approach produces a drag independent of temperature for any crystal structures. Ultrasonic attenuation experiments at low temperatures have confirmed temperature independence of dislocation drag in fec materials, but more precise attenuation experiments are required in bcc materials to produce an experimental comparison of the above two approaches.

The session concluded on the optimistic note that perhaps the time is ripe for at last attempting a rigorous quantum mechanical calculation for the electronic states around a dislocation using such techniques as those recently exploited by Calloway for point defects. 


\subsection{Future Directions for Dislocation Theory}

Panel Members: A. Seeger, N. W. Ashcroft, A. C. Eringen, E. Kröner, J. Lothe, A. A. Maradudin, J. A. Simmons

Questions submitted to the panel were:

1) What more can be done with classical continuum dislocation theory?

a) static $v s$ dynamic formalisms

b) discrete $v s$ continuous distributions

c) linear $v s$ non-linear theories

2) What are the prospects for fruitful interaction between the ideas of dislocation theory and modern continuum mechanics?

3) What is the future for lattice calculations?

a) static theories

b) dynamic theories

4) Are there experimental techniques which can be employed to measure such microscopic phenomena as couple-stresses, non-local effects, disclinations, and incompatibilities, ... perhaps high resolution microscopy, . . .?

5) Can we extend old analogies or uncover new analogies between dislocation theory and other field theories, particularly electromagnetism, hydro-dynamics, general relativity, quantum mechanics...?

6) What are the prospects for our further understanding of thermally activated processes?

7) What quantum effects are likely to be important in dislocation theory? Conversely, what is the hope of doing more meaningful quantum calculations in dislocation theory?

8) Given an interatomic description, what is the best continuum approximation to it? For instance: If a long-range two body potential suffices in the atomic system what is the appropriate continuum formalism? Or how may many body interactions be incorporated into a continuum framework?

9) What are the prospects of producing a macroscopic plasticity theory based on meaningful microscopic parameters? How soon will we be able to contribute to technologically important subjects such as fatigue, fracture, etc.?

The final session of the Conference was devoted to what the Chairman called "futurology."
In dealing with the future of classical continuum dislocation theory the need for more detailed descriptions of dislocation arrangments was strongly brought out. One approach suggested incorporating some of the ideas of statistical mechanics such as higher order correlation functions for the dislocation distribution and/or thermal vibrations, e.g. by means of phonon representations. The advantage of linear (local or non-local) theories over non-linear theories was pointed out-since superposition allows one to use averaged quantities. Also, the power of Green's function techniques were discussed as well as the need for practical calculations of the anisotropic elastic Green's tensors. The importance of the Indenbom-Orlov approach of reducing three-dimensional elasticity problems to two dimensions was questioned and there was the feeling that attempts should be made to use this method. In contrast the defense was made for the use of non-linear continuum theory to treat physical problems where superposition is violated-such as phonon scattering. In addition it was pointed out that higher order elastic constants are being measured experimentally and that it would be worthwhile using them. (One somewhat different application suggested for higher order elastic constants was to get information on pair-forces in metals.)

When the panel turned to question two there was a query from the floor concerning the physical meaningfulness of the "rather tremendous" amount of work going into the continuum theory. One "continuumist" responded with: "There are so-called 'real' theories and 'unreal' theories. Then there are 'really unreal' theories and 'unreal real' theories. Now, continuum theory is the 'really unreal' theory; sometimes it is called 'phenomenological' in order to give an even more deprecative name to it." However, it was agreed that great possibility exists for fruitful interaction between dislocation physics and continuum mechanics. There is a need to directly develop continuum theories which are internally consistent; these theories, which put restrictions on the form of the possible constitutive laws, will aid physicists in finding generalizations from microscopic to macroscopic phenomena. On the other hand it was felt that more dislocation physics should also be incorporated into the already existing theory of plasticity, so that the engineer would have available a better arsenal of practical theory as well as the know-how to make better materials. For instance, it was noted that a real crystal with a static dislocation distribution is very far from thermodynamic equilbrium so that correct thermodynamic approaches are dif- 
ficult. Thus it was concluded that simultaneous development of both approaches was desirable.

Next was the question: What is the future for lattice calculations? This was quickly answered by one panel member: "The future is bright..." The major obstacles to overcome in computerized static lattice calculations are the choice of appropriate boundary conditions and a suitable interatomic potential. Calculations of the change in free energy due to dislocations (for determining Peierl's energy, for instance) and calculation of dislocation-phonon interactions were suggested as feasible research projects.

With regard to experimental techniques for measuring microscopic effects the panel concentrated on the non-local ones. These effects are likely to be important in piezo-electric and ionic crystals; furthermore it was suggested that information about the Fourier transforms of the elastic constants might be obtainable from neutron spectroscopy or from measurements of circularly polarized sound waves in acoustically active crystals.

Finally, turning to question eight, the panel noted that many calculations - such as phonon dispersion-can be carried out in reciprocal space without ever assuming a two-body potential. In fact, it was agreed that even though a two-body potential supplemented by a necessary volume dependent term was useful in many computer calculations, there is no reason for not expecting many-body interactions to be necessary in certain materials, such as some polyvalent metals.

As with the first panel, lack of time necessitated skipping over some questions - in this case questions five, six, seven and nine. It was agreed that question nine was of great technological importance. The session adjourned on the note that "futurology" was by no means exhausted in the two allotted hours.

\section{Conclusion}

This Conference ended with the expressed hope that it would be followed by another such meeting in about three years. Professors Elbaum, Haasen, and Hartley volunteered to pursue this project.

The written proceedings, "Fundamental Aspects of Dislocation Theory," including the contributed papers, discussions, and panels, will be published as NBS Special Publication 317. This Special Publication should appear in the spring of 1970 and will be available from the Superintendent of Documents, Government Printing Office, Washington, D.C. 20402. 


\title{
Nonmetric Connexions, Quasidislocations and Quasidisclina- tions. A Contribution to the Theory of Nonmechanical Stresses in Crystals.
}

\author{
K.-H. Anthony \\ Institut für Theoretische und Angewandte Physik der Universität, Stuttgart, Germany \\ Nonmechanical stresses produced for instance by a temperature gradient or by an internal mag- \\ netic field are related to an affine lattice connexion, which is nonmetric with respect to the elastic met- \\ ric. The covariant derivative of the elastic metric with respect to the lattice connexion is regarded as \\ source-function for this kind of internal stresses. It may easily be determined from experimental data. \\ The calculation of mechanical stresses is based on this quantity. \\ Instead of describing nonmechanical stresses by a nonmetric lattice connexion we alternatively \\ may use an elastic connexion which is metric with respect to the elastic metric. Into the elastic connex- \\ ion there are involved the tensors of quasiplastic torsion and of quasidisclination-density, which are re- \\ garded as source functions of nonmechanical stresses. Both tensors are connected intimately. They are \\ a natural generalization of the quasidislocation tensor in the linear theory of nonmechanical stresses. \\ From the theory of quasidisclinations we obtain the following statement: In general, nonmechanical \\ stresses cannot be eliminated solely by a dislocation movement. An additional distribution of crystal dis- \\ clinations is necessary.
}

\section{One Electron Theories of Cohesion on Ion-Pair Potentials in Metals}

\author{
N. W. Ashcroft \\ Laboratory of Atomic and Solid State Physics, Cornell University, Ithaca, New York 14850
}

The single particle picture of cohesion in metals is briefly reviewed in the light of modern knowledge of their band structures. Periodic components in the electron density distributions (intimately connected with the same band structures) are important in the determination of the effective potential between ions.

In simple metals, defined to be those with tightly bound core states, the net binding of the metallic state is basically a remnant of a competition between kinetic (Pauli principle) and Madelung energies of ostensibly free conduction electrons. Various corrections (for correlation, for exchange, etc.) must be included, and the Madelung energy (which is normally appropriate to a uniform electron gas in a Coulomb lattice of point ions) can be modified as necessary for departures from Coulomb's law. Terms in the total energy also arise from periodic variations in conduction electron density; for perfect lattices these are naturally dependent on the ionic arrangement and will vary in importance from crystal structure to crystal structure. Electronic density variations arising from disorder (e.g. distributions of defects) also introduce corrections into total energy.

The structurally dependent terms in the total energy can be evaluated to second order in the pseudopotential: to this same order the total energy may be written as a sum over pair potentials between ions whose form is quite straight forward to evaluate. As with many inter-atomic potentials, the ion-ion potentials demonstate "hard-core" effects at small separation, and are rather weak at large distances.

Extending the simple theory to transition metals or metals exhibiting additional band structure more akin to itinerant narrow-band behavior, can be carried through by incorporating Born-Mayer interactions between tight binding atomic states. This procedure is, of course, only valid in situations where the Bloch method itself is applicable. While the core-core exchange term approximated by the Born-Mayer interaction is quite small in the simple metals it is appreciable for metals like $\mathrm{Cu}, \mathrm{Ag}$ and $\mathrm{Au}$, and in fact is basically responsible for fixing the equilibrium density. Its addition to the otherwise "simple-metal" like ion pair potentials modifies the behavior at short range. 


\title{
The Dislocation in a Semi-Infinite Isotropic Medium
}

\author{
D. J. Bacon and P. P. Groves
}

\author{
Department of Metallurgy and Materials Science, The University, Liverpool, England
}

\begin{abstract}
The displacement associated with an infinitesimal dislocation loop (displacement dipole) or arbitrary orientation in an isotropic, semi-infinite elastic medium has been obtained, and on integration this yields the displacement associated with a finite dislocation loop. The solution for the infinitesimal loop has been obtained by finding the relationship beiween point forces and displacement dipoles in an infinite medium, and using the same relations with Mindlin's solution for the point force in an elastic half-space. This approach leads to a rather simpler analysis than that of Steketee, and the expressions for the displacements of all the infinitesimal loops near a free-surface are presented here for the first time. The solution is being used to study the stability of a prismatic dislocation loop on a glide prism of square cross-section near a free surface. Preliminary results are presented for the forces on the loop sides and changes in loop energy on rotation for the situation in which the Burgers vector is normal to the surface.
\end{abstract}

\section{Series Representations of the Elastic Green's Tensor for Cubic Media}

\author{
D. M. Barnett
}

\author{
Scientific Research Staff, Ford Motor Company, Dearborn, Michigan 48121
}

Two representations for the cubic Green's tensor components as power series in the anisotropy factor $u=1-\left(c_{11}-c_{12}\right) / 2 c_{4}$ are developed, and first order corrections of anisotropy to the Green's tensor and to the interaction energy between two "mechanical" point defects are calculated. It is shown that the best successive approximation scheme is that which constructs the zeroth order (isotropic) approximation to the Green's tensor by identifying the Lamé constants $\lambda$ and $\mu$ as $\lambda=c_{12}, \mu=c_{44}$.

\section{A Note on Admissible Solutions in Non-Local Elasticity}

\author{
D. M. Barnett
}

Scientific Research Staff, Ford Motor Company, Dearborn, Michigan 48121

In recent years considerable effort has been directed toward incorporating certain features of lattice mechanics within the framework of a continuum description of deforming elastic media. One such theory is non-local elastostatics, developed independently by Kunin, Krumhansl, and Kröner.

The Kunin-Krumhansl non-local scheme is capable of reproducing lattice mechanics exactly by associating a truncated Fourier space with the non-local continuum. The Green's tensor, dislocations, and point defects may be treated rigorously within the framework of this formalism.

Kröner's non-local continuum is associated with a reciprocal Fourier space which need not be truncated. It can be shown that this representation has meaning only if:

(a) The non-local elastic moduli contain a delta function contribution, or, in lieu of this,

(b) The only admissible body force loadings are those which are concentrated over a region larger than that over which the atomic cohesive forces are "appreciable." 


\title{
Generalized Stress and Non-Riemannian Geometry
}

\author{
S. I. Ben-Abraham
}

Instituto Technológico de Aeronáutica CTA, São José dos Campos, S. P., Brazil

The general non-Riemannian approach to the geometry of a solid with lattice defects is briefly discussed. Non-Riemannian geometry is applied to the treatment of statics. The "stress" curvature tensor has the meaning of a generalized stress tensor with 27 components, while the associated Einstein tensor represents the classical stress tensor. The "stress" connexion expresses the generalized moment stress having 27 components. Its torsion tensor is the classical couple stress tensor.

The identities of the curvature tensors are identified with the static equilibrium conditions. Using a stress function density instead of a metric tensor, it is shown that the equilibrium conditions can always be identically fulfilled. A consistent theory involving lattice defects must necessarily involve also generalized moment stresses and generalized stresses.

\section{Kinematics of Continuously Distributed Dislocations}

\author{
S. I. Ben-Abraham
}

\author{
Instituto Technológico de Aeronáutica CTA, São José dos Campos, S. P., Brazil
}

The general non-Riemannian approach to the geometry of a solid with lattice defects is extended to include the kinematics. The treatment is valid for both the motion of old defects and the creation of new ones. The change in a geometric quantity is described by a corresponding rate. The main quantities dealt with are distortion rates, stretching tensors and connexion rates, and new quantities called fluxions are introduced. In terms of these a fundamental kinematic law and continuity equations for the defect currents are set up and are found to be consistent with earlier results valid in special cases.

\section{The Meaning of Dislocations in Crystalline Interfaces}

\author{
W. Bollmann
}

\author{
Battelle Institute, Advanced Studies Center, Geneva, Switzerland
}

\begin{abstract}
The extension of the dislocation concept to arbitrary crystalline interfaces is discussed. It is shown that invariance and continuity of the Burgers vector can be conserved and that in high angle boundaries the function of the standard or primary dislocation is the delimitation of ranges of coordination between the two crystals. In certain relative orientations where the superposition of the two crystals forms a highly periodic pattern (which is energetically favorable such that the crystal tends to conserve it) a slight deviation from that optimum pattern is corrected by a network of secondary dislocations. There is complete balance between the Burgers vectors of primary as well as secondary dislocations.
\end{abstract}




\title{
The Interaction Between a Screw Dislocation and Carbon in Body-Centered Cubic Iron According to an Atomic Model
}

\author{
Roger Chang
}

Science Center, North American Rockwell Corporation, Thousand Oaks, California 91360

The interaction energy between carbon and a screw dislocation in body-centered cubic iron near the core regions of the dislocation was calculated atomistically using a pair-wise interatomic potential matching the elastic properties of the material. In order to avoid the use of the iron-carbon potential, it was assumed that the iron-carbon octahedron of the Johnson configuration ( 2 iron atoms separated by $1.225 a_{0}$ in the [100] direction and 4 iron atoms separated by $0.958 a_{0}$ in the (100) plane, $a_{0}$ being the lattice parameter) remains undistorted whether it is present in a perfect or a defective lattice. Our first calculations yield, depending on site location, binding energies varying from 0.03 to $1.96 \mathrm{eV}$. The binding energy is sensitive to changes in hydrostatic pressure and the estimated volume dependence offers a convenient interpretation of the strength-differential effect of martensitic steels via the nonlinear carbon-dislocation interaction mechanism first proposed by Hirth and Cohen.

\section{Strain Rates in Dislocation Dynamics}

\author{
W. deRosset and A. V. Granato \\ Department of Physics, University of Illinois, Urbana, Illinois 61801
}

Since the success by Johnston and Gilman in relating yield phenomena in LiF to direct measurements of dislocation velocities and densities. the $\Lambda b v$ expression for the strain rate has been used almost exclusively, where $\Lambda$ is the mobile dislocation density, $b$ the Burgers vector, and $v$ is the average dislocation velocity. We note that there are cases for which the strain rate is independent of dislocation velocity. When the velocitv is large enough the strain rate is given in a simplified model by $N b \bar{x}$, where $\bar{x}$ is the average distance traveled by a dislocation. The strain rate is eventually determined, then, by the rate at which new dislocations are created and not necessarily by the rate at which old dislocations expand or by the dislocation velocity.

\section{Linear Theory of Static Disclinations}

\author{
Roland deWit
}

Metallurgy Division, National Bureau of Standards, Washington, D.C. 20234

\begin{abstract}
A brief review of compatible and incompatible elasticity theory is given. It is shown how dislocation theory was developed from classical compatible elasticity. Then disclination theory is developed from dislocation theory in an analogous way. The disclination and dislocation density tensors are defined from the plastic deformation. The total deformation satisfies the classical condition of compatibility. By combining these two concepts the geometric basic laws or field equations are found, which relate the elastic deformation to the defect content. The contortion or Nye curvature tensor is found to be a useful equivalent for the dislocation density. Weingarten's theorem motivates the generalization of the Burgers vector and the definition of the analogous rotation vector of disclinations. Finally the dualism between the geometry of disclination theory and the statics of couple-stress theory, and the relation of disclination theory to "motor calculus" are pointed out.
\end{abstract}




\title{
Atomistic Calculation of Dislocation in Solid Krypton
}

\author{
M. Doyama
}

Argonne National Laboratory and University of Tokyo, Japan

and

\section{R. M. J. Cotterill}

Argonne National Laboratory and The Technical University of Denmark, Lyngby, Denmark

The elastic continuum theory treatment usually fails near the core of dislocations. Atomic calculations of edge and screw dislocations in solid krypton were performed very successfully using a pairwise potential. In rare gases, the electron redistribution of the electron density is small, thus, this method is useful to study the properties concerning the core of dislocations.

\section{On the Mechanism of Cross-Slip of Dislocations at Particles}

\author{
M. S. Duesbery* and P. B. Hirsch \\ Department of Metallurgy, University of Oxford, England
}

\begin{abstract}
The effect of the misfit strain field upon the mechanism of cross-slip of dislocations at impenetrable particles in two-phase alloys is considered. The strain field is found to have a profound effect of the activation energy for cross-slip.
\end{abstract}

\section{The Anharmonic Properties of Vibrating Dislocations}

\author{
C. Elbaum and A. Hikata \\ Department of Physics, Brown University, Providence, R.I. 02912
}

\begin{abstract}
The anharmonic properties of vibrating dislocations are discussed in terms of the nonlinear stress-strain relation and of the higher harmonics of an ultrasonic wave generated when an initially sinusoidal wave propagates in a solid containıng (mobile) dislocations. The treatment takes account of both lattice and dislocation contributions to the anharmonic behavior of the solid.

Estimates of the amplitude of the harmonics (these estimates have been confirmed experimentally) indicate that the lattice and dislocation components are comparable for the second harmonic and that the dislocation component is much larger than the lattice component for the third harmonic. Therefore, by investigating the third harmonic, it is possible to obtain detailed information on dislocation dynamics, without the complications of the lattice contribution.
\end{abstract}




\title{
Interactions of Dislocations with Electrons in Metals
}

\author{
C. Elbaum and A. Hikata
}

Department of Physics, Brown University, Providence, R.I. 02912

\begin{abstract}
Physical arguments are given to show that the interaction of moving (vibrating) dislocations with conduction electrons in metals is independent of temperature. Calculations based on the assumption that the Fourier components of the displacement field of a moving dislocation can be treated as pseudo-phonons give the same result. These conclusions were confirmed by the results of recent experiments.
\end{abstract}

\section{Electronic Energy States of Dislocations; The Case of Covalent-Ionic Solids}

\author{
C. Elbaum and R. R. Holmes* \\ Department of Physics, Brown University, Providence, R.I. 02912
}

\begin{abstract}
It is shown that electronic energy bands are associated with dislocations in wide-band-gap, com pound semiconductors. The eigenvalue problem for the dislocation band edge is solved for CdS-type crystals, and the occupanon of the band is calculated. The Fermi energy is then determined for crystals containing many deep-lying discrete levels as well as dislocation bands. It is predicted that when a crystal is illuminated with light of appropriate wavelength and increasing intensity, the thermal activation energy governing the electrical conductivity passes through a series of energy plateaus which are equal to the energy of the discrete levels.In a dislocation-free crystal, these plateaus are connected by step changes, while in a crystal with dislocations they are connected by broad transition regions. The above predictions have been verified experimentally.
\end{abstract}

\section{Theories of Thermally Activated Processes and Their Application to Dislocation Motions in Crystals}

\begin{abstract}
H. Engelke
Institut für Theoretische und Angewandte Physik der Universität, Stuttgart, Germany

Thermally activated processes involving dislocations are usually treated by applying the theory of absolute reaction rates and the string approximation of dislocation. In the first part of this paper the limits of validity of these treatments are discussed. A paper of T. Geszti is briefly reviewed in which it is shown that in processes involving point defects deviations from the rate theory must be expected if the energy exchange between defect and lattice waves is slow compared with the average time between jumps, e.g. if localized of quasi-local vibrations contribute essentially to the defect motion. It is pointed out that the localized modes of dislocations may also lead to deviations from the rate theory.

In the second part a treatment of the double kink relaxation (Bordoni relaxation) based on the Kramers-Brinkman diffusion theory of chemical reactions is given. After showing that in f.c.c. metals the influence of the Peierls potential is so small that on dislocation loops only one kink pair must be con-
\end{abstract}

*Present address: Bell Telephone Laboratories, Whippany, N.J. 
sidered explicitly whereas the remaining parts of the loops may be described by the string approximation diffusion equations for the loop motion are derived. Under certain conditions these equations are equivalent to a system of reaction rate equations. Exact solutions of this system are not yet available. However, the behaviour of dislocation loops in the amplitude independent region of internal friction measurements may be extrapolated combining results of two limiting cases: a double well model and a simplified multi-well model.

The relaxation strength and the preexponential factor of the relaxation time calculated have the same orders of magnitude as in measurements of the Bordoni peak. The loop length dependence of the damping peak is in good agreement with neutron-irradiation measurements. The characterisstic half-width of the Bordoni peak may partly be explained using loop length and stress distributions.

\title{
Point Defects and Dislocations in Copper
}

\author{
A. Englert,* H. Tompé \\ Union Carbide European Research Associates, 95, Rue Gatti de Gamond, Brussels 18, Belgium \\ and \\ R. Bullough \\ Theoretical Physics Division, A.E.R.E., Harwell, Didcot, England

\begin{abstract}
A new pair potential for copper has been constructed from a set of ten interpolated cubic polynomials. The form of the potential is such that at short range it agrees with the usual Born-Mayer repulsive potential and is in satisfactory agreement with the available phonon dispersion data and the observed stacking fault energy and vacancy formation energy for copper. The potential has been used to study the atomic configuration associated with various point and line defects in copper. In particular, because of its fit to the stacking fault energy, it provides a consistent result for the degree and nature of the dissociation to be expected for an edge dislocation in copper.
\end{abstract}

\section{A Micromorphic Approach to Dislocation Theory and its Relation to Several Existing Theories}

\author{
A. C. Eringen \\ Princeton University, Princeton, New Jersey 08540 \\ and
}

W. D. Claus, Jr.

Princeton University and Textile Research Institute, Princeton, New Jersey 08540

\begin{abstract}
Two separate continuum dislocation theories are presented; one dealing with static, incompatible, micropolar dislocations and disclinations, as encountered in initial stress problems, and the other with a dynamical theory of micromorphic solids containing continuous distributions of dislocations.

Relationships between several continuum dislocation theories and micromorphic mechanics are established by providing extensions and new interpretations of the micromorphic theory. First both micromorphic and micropolar theories of elastic solids are summarized, and then the theories of Kröner, Fox, and Berdichevskii and Sedov are discussed in some detail within this framework. In the last section, by use of micromorphic kinematics, dislocation density, strain, and microstrain tensors are introduced and constitutive equations are constructed. Together with the balance laws this constitutes a complete dynamical theory. The theory is intended for predictions of motions and micromotions of a solid containing dislocations undergoing elastic deformations. From the micromotion, the dislocation density and first stress moments can be calculated.
\end{abstract}

*Visiting Research Associate, Harwell, Summer 1968 


\title{
The Method of Lattice Statics
}

\author{
J. W. Flocken and J. R. Hardy
}

Behlen Laboratory of Physics, University of Nebraska, Lincoln, Nebraska 68508

The formalism of the method of lattice statics for treating the lattice distortions and the formation and interaction energies associated with a defect in a crystal is presented in detail. This approach is based on the Fourier transformation of the set of direct space equilibrium equations to reciprocal space. This results in a set of decoupled equations which can be explicitly solved for the Fourier amplitudes of the displacement field which can then be found by Fourier inversion. A similar approach is used to obtain Fourier transformed expressions for the relaxation and interaction energies associated with the defect.

The solution of the equations of lattice statics for the Fourier amplitudes in the limit of small wave vectors gives expressions for the displacement field identical to those obtained from the theory of continuum elasticity.

Results are presented of recent applications of the method of lattice statics to find the formation: energies of Schottky pairs in certain alkali halides. Strain field displacements, relaxation energies and interaction energies associated with vacancies in $\mathrm{Na}$ and $\mathrm{K}$ are given.

Lattice statics in its asymptotic form has been used to find the displacement field far from cubic point defects and double defects in a number of metals. Displacement profiles about vacancies in $\mathrm{Na}$ and $\mathrm{K}$ and about a double force defect in $\mathrm{Cu}$ are shown. A comparison of the exact lattice statics results to asymptotic results along the $\langle 111\rangle$ direction in $\mathrm{K}$ shows that the elastic limit is only attained at about the 19th or 20th neighbor position from the defect.

\section{Movement of a Dislocation Through Random Arrays of Point and Parallel Line Obstacles}

\author{
A. J. E. Foreman \\ A.E.R.E., Harwell, Didcot, England \\ and \\ P. B. Hirsch and F. J. Humphreys \\ Department of Metallurgy, University of Oxford, England

\begin{abstract}
The motion of a dislocation through random arrays of impenetrable point and parallel line obstacles is considered. For impenetrable point obstacles the difference in line tension for edges and screws, and the elliptical equilibrium shape of the bowing-out loop are taken into account, and the yield stress for screws and edges is found to be the same. The results of a calculation of the effect of interaction between neighboring segments of the dislocation on the critical breaking angle and on the yield stress are presented. Computer calculations on the motion of dislocations through random arrays of parallel line obstacles show that the dislocations move by the generation and motion of large "kinks." The yield stress is found to increase approximately linearly with the obstacle length. These calculations are relevant to and are compared with the hardening in the early stages of the work hardening curve of dispersion hardened alloys containing small particles, in which rows of prismatic loops are formed at the particles, which act as linear obstacles to the gliding dislocations.
\end{abstract}




\title{
On the Continuum Theory of Dislocations
}

\author{
N. Fox \\ Department of Applied Mathematics, University of Sheffield, England
}

The kinematics of an oriented medium is discussed with reference to the contiuum theory of dislocations. The concepts of dislocation density, dislocation flux and slip velocity are introduced and a corresponding constitutive theory is developed.

\section{Dislocation Dynamics in the Presence of a Multiple Spectrum of Thermally Surmountable Barriers}

\begin{abstract}
W. Frank
Max-Planck-Institut für Metallforschung, Institut für Physik, Stuttgart, Germany

A theoretical treatment is given of the thermally activated motion of dislocations in the presence of dislocation barriers with different heights. The height of a barrier is allowed to depend on an arbitrary number of characteristic parameters (multiple obstacle spectrum). Under the assumptions which are usually made for the derivation of an Arrhenius equation for the slip rate $\dot{a}$ in the case of a uniform array of dislocation barriers, $\dot{a}$ is calculated for a multiple spectrum of barriers. It is found that even under these conditions the validity of an Arrhenius equation $\dot{a}=\dot{a}_{0} \exp (-\overline{\Delta G} / k T)\left(a_{0}=\right.$ const., $\overline{\Delta G}=$ mean enthalpy of activation) is preserved. An application of the "spectrum theory" is discussed.
\end{abstract}

\section{Dislocation Resonance}

\author{
J. A. Garber and A. V. Granato \\ Department of Physics, University of Illinois, Urbana, Illinois 61801
}

\begin{abstract}
At low temperatures in insulators and superconductors, only reradiation of elastic waves should limit dislocation resonance. This effects has been calculated using Eshelby's expression for the reradiation by analogy with radiation damping effects in the electromagnetic case. It is found that the resonance is very sharp, and still persists even when a random distribution of dislocation segment lengths is assumed.
\end{abstract}

\section{The Structure of the $\left\langle\begin{array}{lll}1 & 1\end{array}\right\rangle$ Screw Dislocation in Iron}

\author{
P. C. Gehlen, G. T. Hahn, and A. R. Rosenfield \\ Metal Science Group, Battelle Memorial Institute, Columbus, Ohio 43201
}

The concept of a dissociated a/2 $\langle 111\rangle$ screw dislocation has been invoked to explain the slip behavior in bcc materials and particularly the asymmetry of the critical resolved shear stress. No direct experimental evidence of dissociation has been obtained, but the idea has received some albeit conflicting support from discrete lattice calculations of the atomic positions in the core. Chang, using isotropic elasticity for $\alpha$-iron, found that the dislocation core has three very narrow intrinsic faults. These 
three faults are symmetric with respect to the screw axis. Bullough and Perrin, on the other hand, found that the screw is split with faults on two $\{112\}$ planes belonging to the zone of the screw axis. The misfit is spread over a distance of about $3 b$. On the third $\{112\}$ plane no splitting was found to occur.

In view of these discrepancies, the calculations were repeated for anisotropic and isotropic elastic boundary conditions and with different interatomic potentials. Excellent agreement was found with Chang's configuration even though a volume expansion term was added to the displacements associated with the dislocation.

It was shown that the final configuration is strongly dependent on the position of the dislocation line with respect to the lattice and at least two metastable positions were found. Even though the atomic arrangment is quite different, their energy is not more than $0.1 \mathrm{eV}$ larger than the energy of the stable one.

Using the Johnson potential unmodified for long-range electronic effects, the dislocation was found to have the following characteristics: core radius, 4-5.5 $\AA$; core energy, $0.20-0.25 \mathrm{eV}$ per atomic plane; and an effective hole radius of $1.35 \AA$.

It was shown that the final configurations are rather insensitive to the model size and to the boundary conditions used.

\title{
Phonon Scattering by Dislocations and its Influence on the Lattice Thermal Conductivity and on the Dislocation Mobility at Low Temperatures
}

\author{
P. P. Gruner
}

Boeing Scientific Research Laboratories, P.O. Box 3981, Seattle, Washington 98124

On account of the large strains associated with dislocations, the superposition principle is violated. The resulting scattering of phonons limits the lattice thermal conductivity and leads to a friction force which acts on moving dislocations. The phonon-dislocation interaction is treated with non-linear continuum theory. Terms up to the third order in the strains are retained in the Taylor expansion of the elastic energy density. These third order terms contain the phonon-dislocation interaction and the normal three-phonon interactions. In the case of thermal conductivity, the transport problem is solved with the variational method which leads to a system of linear equations for the phonon occupation numbers. The coefficients of this system of equations contain all the information on the scattering mechanisms. The influence on the thermal conductivity of special dislocation configurations such as piled-up dislocations and dislocation dipoles will be discussed.

It will be shown that the friction force which acts on moving dislocations on account of the anharmonicity can be obtained from quantities that are known from the calculations of the phonon conductivity. A one to one correspondence between friction force and thermal resistance exists, however, only if the dislocation velocity is small compared with the sound velocity and if all parts of the dislocation move with the same velocity.

\section{Charged Dislocations in the Diamond Structure}

\author{
P. Haasen and W. Schröter \\ Institut für Metallphysik, Universität Göttingen, Germany
}

\begin{abstract}
The electronic states at dislocations in elemental semiconductors are reviewed in the light of new experimental and theoretical work. Evidence is produced for the existence of a one-dimensional energy band along edge as well as screw dislocations. This explains quantitatively their ability to act as donors as well as acceptors depending on temperature, doping, and dislocation density. The degree of charging has been recalculated from the free energy of a system of charged dislocations screened by free carriers. The dislocation charge limits the Hall mobility in addition to the strain field of the dislocation. Measurements of photo conductivity of deformed semiconductors support the above model of the charged dislocation. The dislocation charge influences the Peierls force and thus the mobility of dislocations in the diamond structure. The occupation limit of the neutral dislocation, measured from the valence band edge, lies at $0.09 \mathrm{eV}$ in germanium, and at $0.3 \mathrm{eV}$ in silicon.
\end{abstract}




\title{
Toplogical Restriction on the Distribution of Defects in Surface Crystals and Possible Biophysical Application
}

\author{
W. F. Harris \\ Department of Chemical Engineering, Institute of Technology, University of Minnesota, \\ Minneapolis, Minnesota 55455

\begin{abstract}
Many thin biological structures such as some plasma membranes and virus capsids appear to be made up of units packed in two-dimensional lattices. Such structures are termed surface crystals. Dislocations and disclinations are observable in some of these crystals. The perfect surface crystal is described by a pair of basic vectors and the conventional crystals by a triplet of basis vectors; both are regarded as embedded in three-dimensional space. This difference allows the existence of defects which have no counterpart in conventional crystals. The various defects are classified as local or global, intrinsic or extrinsic. Surface crystals that form closed surfaces are considered and it is shown that the sum of the rotation of intrinsic (screw) disclinations in them must equal $2 \pi \chi$ where $\chi$ is the Euler-Poincaré characteristic of the surface. The biophysical consequences are discussed briefly.
\end{abstract}

\section{Elastic-Plastic Plane Bending of a Single Crystal}

\author{
C. S. Hartley and M. A. Eisenberg
}

Department of Engineering Science and Mechanics, University of Florida, Gainesville, Florida 32601

The elastic-plastic bending of a single crystal beam which deforms by single slip on a plane containing the axis of bending and in a direction normal to the axis of bending is analyzed. Both continuum plasticity and dislocation approaches are used to obtain stresses and/or displacements in the elastic and plastic regions. The relation between the applied bending moment and the curvature of the neutral axis is also obtained. The approaches are compared and correspondences between the two theories are discussed.

\section{Discrete Dislocations in Continuum Elasticity}

\author{
A. K. Head \\ Commonwealth Scientific and Industrial Research Organization, Division of Tribophysics, \\ University of Melbourne, Victoria, Australia
}

As a framework in which to survey some of the current questions in this long-established part of dislocation theory, the problem of the experimental determination of stacking fault energy is considered. Attention is restricted to those methods which observe and measure in the electron microscope a simple dislocation-stacking fault configuration. It is assumed that the configuration is in equilibrium with the forces between the dislocation balancing the surface tension of the stacking fault. The stacking fault energy then follows if these dislocation forces are known from theory for the observed dimensions of the configuration.

Experimental techniques have improved to the point where a reproducibility of 1 to 5 percent standard deviation is claimed. This calls for theory which has at least a similar standard of accuracy. It is doubtful if the theory which is in current use has this accuracy and this is surveyed under the following headings: a) The treatment of the core; b) Elastic anisotropy; c) Zig-zag instabilities; d) The effect of the stacking fault; e) The observation of dislocations by electron microscopy. 


\title{
Slightly Dissociated Dislocations
}

\author{
R. Hobart
}

Battelle Memorial Institute, Columbus Laboratories, 505 King Avenue, Columbus, Ohio 43201

A modified Peierls-Nabarro model is used to calculate the relation between the separation of partials and stacking fault energy for an idealized dislocation dissociated into two equal pure screw partials. The resulting expression agrees with the inverse dependence obtained by equating the elastic repulsion of the partials to the attraction of the stacking fault, but unlike that result does not break down at zero separation. Also an argument is given that the width of such a dissociated dislocation should be considered as a complex quantity, with the imaginary part corresponding to the separation of the partials.

\section{Thermodynamic Properties of Solids Containing Dislocations}

\author{
J. Holder and A. V. Granato \\ Department of Physics, University of Illinois, Urbana, Illinois 61801
}

\begin{abstract}
Using thermodynamics and elasticity theory, a unified treatment of the changes in the thermodynamic properties of solids containing dislocations is given. The results are expressed in terms of temperature and pressure derivatives of the formation energy of the dislocations. The required pressure and temperature dependence of the energy is given by the measured pressure and temperature dependence of the elastic constants appearing in the energy expression found at zero pressure and temperature. The method is not restricted to calculations of volume changes, or to isotropic, static or even elastic systems at zero pressure. Changes in entropy, specific heat, thermal expansion and bulk modulus due to dislocations are easily calculated.
\end{abstract}

\section{A Lattice Theory Model for Peierls-Energy Calculations}

\author{
A. Hölzler and R. Siems
}

\author{
Institut für Theoretische Physik C der Technische Hochschule, Aachen, Germany
}

\begin{abstract}
A lattice theory model for a screw dislocation is discussed which is similar to that of Maradudin. For the forces between neighbouring rows of atoms, however, a sinusoidal, not a linear, dependence of their relative displacements is assumed throughout the whole lattice. The displacements are expanded about the elastic theory values. The conditions of equilibrium then yield a system of linear equations for the deviations of the displacements from the elastic theory values, which is solved by an iteration procedure making use of Green's Function for a plane square lattice. For a number of points in the vicinity of the source point and for points in certain symmetry directions simple exact analytical expresssions for the latter are derived, for points at larger distances an asymptotic expansion is given. The displacements thus obtained are then used to calculate the energies of the dislocation at the position of minimum energy and at the saddle point and their difference, the Peierls energy, by direct summation of the interaction energies of neighbouring pairs of atoms.
\end{abstract}




\title{
Interactions Between Electrons and Moving Dislocations
}

\author{
G. P. Huffman and N. Louat,
}

\author{
Edgar C. Bain Laboratory for Fundamental Research, U.S. Steel Corporation Research Center, \\ Monroeville, Pennsylvania 15146
}

\begin{abstract}
A moving dislocation in a metal induces an electric field which causes currents in the conduction electron gas. The energy dissipation associated with these fields and currents is calculated from the Boltzmann equation in a manner analogous to theories of acoustical attenuation. It is found that the applied stress required for steady motion is proportional to the dislocation velocity divided by the electrical resistivity, in good agreement with low temperature yield and flow stress measurements on bcc metals. The concept of a large electronic component of drag which increases with decreasing temperature is used to give a qualitative explanation of the observed mechanical properties of bbc metals. When one considers electronically damped dislocations impeded by pinning points, it is found that: 1) the dislocation velocity depends only on the mean strength of the pinning obstacles; 2) the stress required to overcome an obstacle dynamically is less than that required statically and is, in fact, the electronic drag stress; and 3) the apparent dislocation velocity should vary rapidly with applied stress. The displacement field of a dislocation is significantly wider and more gradual in a fcc than in a bec lattice, and this feature can be incorporated into the calculation by assuming that the electronic screening of the positive charge shift of the dislocation is essentially perfect in a fcc lattice. The Boltzmann formation then gives a temperature independent stress or drag coefficient in agreement with experiment and with Holstein's scattering calculation. The problem of a dislocation moving in an applied magnetic field directed along its length and perpendicular to its direction of motion is also treated. Under suitable conditions it is found that the stress or drag coefficients exhibit oscillations of the cyclotron resonance type which could be as large as 10 to $30 \%$ of the zero field values.
\end{abstract}

\section{Identification of the Dragging Mechanisms of Dislocations}

\author{
V. L. Indenbom \\ Institute of Crystallography, Academy of Sciences, Moscow, USSR \\ and
}

A. N. Orlov

Physico-Technical Institute, Academy of Sciences, Leningrad, USSR

\begin{abstract}
The results of direct investigations of the dislocations movement makes it possible to proceed to identification of the mechanisms, controlling mobility under different conditions and require some revision of theoretical estimations of various mechanisms contribution.

Investigations of high-speed dislocations and amplitude independent internal friction yield data concerning the intensity of energy dissipation which can be explained by the transmission of an impulse from the dislocation to different branches of the spectrum of elementary excitation of the crystal: phonons, electrons, exitons and others.

Most reasonable estimations result from computations of the dynamic Peierls force. In case of even motion of dislocations certain phonon mechanisms do not provide the observed dissipation rate. The accord between theory and experiment improves to a greater extent if the oscillations of the dislocation field due to its movement across the Peierls relief are taken into account.

As temperature falls phonon processes are frozen out and electron mechanisms of dissipation are displayed. Their contribution is clearly exposed in case of superconductors.

Excitations of internal degrees of freedom of a dislocation corresponds to another class of the dragging mechanisms. Creation, movement and interaction of kinks, jogs and other elements of the fine structure of the dislocation core account for microgeometry of a dislocation and its dynamic properties. Elementary mechanisms of this kind are observed in the spectors of internal friction and can be analysed on the basis of the latest observations of dislocation mobility in semiconductors.
\end{abstract}




\title{
Aspects in Liquid Crystals Relevant to the Theory of Dislocations
}

\author{
M. Kléman
}

Laboratoire de Physique des Solides, Faculté des Sciences, 91 -Orsay, France

A general theory of dislocations in a mesomorphic medium is outlined. On the basis of their symmetry properties, and of viscosity relaxation, it is first shown that perfect disinclinations in nematic crystals may take any shape, are restricted to straight lines in smectic crystals, and that both types exist in cholesteric crystals, where there are two possible axes of rotation. The second part is specialized to the case of cholesteric crystals, for which a mechanism of pairing of dislocations is proposed which allows a multiple disinclination to take any shape, at the expense of a small increase of energy in the faulted ribbon. The observed magnetic instability of these pairs, which constitutes a strong evidence of the proposed model, is explained.

\section{Phonon Scattering by Cottrell Atmospheres}

\author{
P. G. Klemens
}

Department of Physics and Institute of Materials Science, University of Connecticut, Storrs, Connecticut 06268

The formation of Cottrell atmospheres can change the scattering of phonons by dislocations and in some cases substantially enhance the lattice thermal resistivity due to dislocations. The strength of the atmospheres can be changed by annealing. This changes thermal conductivity values at high temperatures first, since diffusion through shorter distances is involved. The diffusion coefficient can be determined by means of such annealing studies.

\section{Flow and the Arrhenius Equation in the Statistical Framework}

\author{
U. F. Kocks
}

\author{
Metallurgy Division, Argonne National Laboratory, Argonne, Illinois 60439
}

\begin{abstract}
The rate equation of plastic flow and a general work hardening law are combined into a single equation of flow. It degenerates into the Arrhenius law only under certain specified conditions. A single Arrhenius term with temperature insensitive pre-exponential (and entropy) terms adequately describes the rate dependence of dislocation motion through a slip plane studded with obstacles of any spectrum of strengths and spacings. In this case, the mobile dislocation density in the slip plane generally does not enter the flow equation; only the density of slip planes does.
\end{abstract}

\section{A Non-Riemannian Construction of Variational Criteria for Plastic Manifolds with Special Reference to the Theory of Yielding}

\author{
Kazuo Kondo \\ University of Tokyo, Tokyo, Japan
}

The variational formalism is viewed not as an independent assumption but as a natural consequence following from a more fundamental penetration into the recognition of nature and this is shown in regard to the static aspects of plasticity. The energy is defined as the necessary quantity for the geometrical construction. With the Riemannian and non-Riemannian picture, a bridge is sought between the theory of yielding and the theory of dislocations, as also between the theory of dual yielding 
and theory of couple stresses. The standpoint of the theory of yielding and dual yielding is explained constitutively with a statistical construction to reconfirm the formulae which have been proposed. The meaning of the plastic constants involved in the theory is clarified by the construction.

\title{
The Problem of Non-Locality in the Mechanics of Solids: Review on Present Status \\ E. Kröner
}

\author{
Institut für Theoretische Physik, Technische Universität, Clausthal, Germany \\ The concept of non-locality, in particular with reference to the mechanics of solids, is discussed. A \\ brief report is given on the derivation of the non-local theory of elasticity from atomic lattice theory. In \\ addition some simple solutions of this theory, concerning the interaction of dilatation centers and wave \\ propagation, are quoted. A generalization of the theory which would include dislocations is indicated.
}

\section{Non-Local Theory of Elasticity for a Finite Inhomogeneous Medium - A Derivation from Lattice Theory}

\author{
E. Kröner and B. K. Datta
}

Institut für Theoretische Physik, Technische Universität, Clausthal, Germany

In the linear theory of non-local elasticity the strain energy can be written as a double volume integral summing up the interactions of pairs of mass elements. The properties of the material, in this case, are described by a two-point-tensor function $c_{i j k l}\left(\mathbf{r}, \mathbf{r}^{\prime}\right)$. It is found that for finite bodies $c_{i j k l}\left(\mathbf{r}, \mathbf{r}^{\prime}\right)$ does not have the symmetries of the (Hooke's) elasticity tensor $C_{i j k l}$ of the local theory. However, the symmetries of this tensor function are sufficient to exclude the rotations from the strain energy expression. It is shown that with the help of the elastic Green's function one can express $c_{i j k l}\left(\mathbf{r}, \mathbf{r}^{\prime}\right)$ in terms of force constants of the lattice. It is also shown that an infinite homogeneous body is not a suitable model either for the non-local or for the local theory, because in this case $c_{i j k l}\left(\mathbf{r}, \mathbf{r}^{\prime}\right)$ as well as $\mathrm{C}_{i j k l}$ obey the Cauchy relations.

\section{Theoretical Considerations on the Extension of $1 / 2\langle 110\rangle$, $\left\{\begin{array}{lll}1 & 1\end{array}\right\}$ Dislocations in Isotropic FCC Metals into Shockley Partials}

\author{
Doris Kuhlmann-Wilsdorf
}

Department of Materials Science, University of Virginia, Charlottesville, Virginia 22903

and

\section{T. R. Duncan}

Department of Minerals and Metals Engineering, University of Wisconsin, Madison, Wisconsin 53706

The energy changes and equilibrium separations associated with the extension of $1 / 2\langle 110\rangle,\{111\}$ dislocations in fec metals into Shockley partials have been calculated and were found considerably smaller than the previous corresponding values due to Seeger and Schöck. The new results may be shown to be credible on the basis of the following simple estimates. 
The theoretical shear fracture strength of a crystal may be written as $\tau_{\text {crit }}=G / q$, with $G$ the appropriate shear modulus and the numerical parameter $q$ near 30 for $\{111\}$ planes in fec metals. The resolved shear stress required to force the Shockley partials into coincidence at vanishing stacking fault energy, $\left(\tau_{e q}\right)_{\max }$, must approach but cannot exceed $\tau_{\text {crit }}$ so that $\left(\tau_{e q}\right)_{\max } \equiv G / 30$, i.e. much smaller than the value of $\sqrt{6} G / 8 \pi \simeq G / 9$ previously assumed. Similarly, the stacking fault energy cannot exceed the value of $\gamma_{c r i t}=G a / \sqrt{6} q$ with $a$ the lattice constant, and the dislocation core energy must be in the order of $E_{c} \simeq 2 r_{r} \gamma_{c r i t} \simeq 1 / 4 E_{D}$ where $E_{D}$ is the total dislocation energy, and the core radius, $r_{0}$, is taken to be that distance from the dislocation axis at which the resolved shear stress on the slip plane would become equal to $\tau_{\text {crit }}$ in linear elastic behavior. Lastly, $D_{0}$, the actual equilibrium separation between the partials must be considerably smaller than $D_{0}{ }^{*}$, the separation calculated from linear elastic theory, as long as $D_{0}{ }^{*}<2 r_{0}$. One finds easily that $D_{0}{ }^{*} \leqslant 2 r_{0}$ for $\gamma \geqq \gamma_{\text {crit }} / 6$ and $\gamma \gtrsim \gamma_{\text {crit }} / 10$ for edge and screw dislocations, respectively, depending somewhat on Poisson's ratio, so that only in silver will edge dislocations have an equilibrium separation a little larger than $2 r_{0}$, but much less than $2 r_{0}$ in the other common pure fcc metais. For $D_{0}=2 r_{0}$, when the core energy is reduced by about $1 / 3$ according to the rule of the square of the Burgers vectors but the elastic energy is virtually unaffected, and remembering that work $2 r_{0} \gamma$ with $\gamma \simeq \gamma_{\text {crit }} / 6$ must be done to spread out the stacking fault, the relative energy gain due to the extension of edge dislocations is simply estimated as $\left(\Delta E / E_{D}\right)_{\perp} \simeq\left[1 / 3 E_{c} / 4 E_{c}-1 / 6 \gamma_{\text {crit }} / 4 \gamma_{\text {crit }}\right] \simeq 4 \%$ which may be compared to the calculated value of $4.6 \%$ in silver.

\title{
On Problems of the Non-Local Theory of Elasticity
}

\author{
I. A. Kunin and A. M. Waisman \\ Institute of Thermophysics, Academy of Sciences, Novosibirsk, USSR
}

The paper contains new results concerning the linear theory of an elastic medium with non-local interactions:

I. The structure of the general solution of the equations of motion. The dependence of the general solution on the distribution of energy operator zeroes. Specific effects of the non-locality: new types of dampled and undamped waves, the existence of a maximum frequency for the latter ones, the space dispersion. The Green's function.

II. Approximate models. Two types of approximate models: the long-wave approximations and the first zeroes approximations. Domains of their applicability.

III. Boundary value problems. The replacing of the boundary by a boundary layer of width of the interaction radius. Generalized Green's formula. Fundamental boundary value problems, their correct formulation for first zeroes approximations. The comparison with couple-stress theories. Examples of exact and approximate solutions. The existence of new types of surface wave different from Rayleigh waves.

\section{Some Recent Results on Dislocation Pileups}

\author{
J. C. M. Li \\ Materials Research Center, Allied Chemical Corporation, Morristown, New Jersey 07960 \\ A few results intended to illustrate the usefulness of orthogonal polynomials and singular integral \\ equations for the problem of dislocation pileups are described. A simple method of solving a few special \\ integral equations is suggested. The usefulness of a Moutier cycle for the calculation of stress concen- \\ tration is shown.
}




\title{
The Image Force on Dislocations at Free Surfaces. Comments on the Concept of Line Tension.
}

\author{
J. Lothe \\ Institute of Physics, University of Oslo, Blindern, Oslo, Norway
}

\begin{abstract}
It is shown that a straight dislocation emerging at a planar free surface is acted upon by a force distributed according to the law $d \mathrm{~F}=\lambda^{-1}(-E \operatorname{cotan} \theta+\partial E / \partial \theta) d \lambda$. Here $\theta$ is angle of inclination to surface, $E$ is dislocation energy factor, and $\lambda$ is distance from surface along the dislocation. The proof involves use of a slightly generalized version of the energy flow theorem for straight dislocations (Lothe, Phil. Mag. 15, 9 (1967)). The above formula is the exact linear elasticity law corresponding to the usual approximate line tension result. According to this law there are certain angles of incidence for which the forces vanish so that the dislocation can be straight. Similarly, when only elastic terms are considered, there are certain node orientations for which the forces on the branches vanish so that the branches can be truly straight (Indenbom and Dubnova, Sovj. Phys. (Solid State), 9, 915 (1967)). In this paper a discussion is given of how core energy terms may modify the results. It is concluded that also for nodes in the characteristic orientation for no elastic forces on the branches, some branch curvature will be present near the node.
\end{abstract}

\section{Some Problems Involving Linear Dislocation Arrays}

\author{
N. Louat \\ Edgar C. Bain Laboratory For Fundamental Research, U.S. Steel Corporation, Research Center,
Monroeville, Pennsylvania 15146

\begin{abstract}
Muskhelishvili's inversion formulae for singular integral equations are shown to be special cases of a more general result which is then employed to deal with two types of problems. In the first we consider the distribution of dislocations in a double periodic array of screw pile-ups in an arbitrary stress field. The second type is concerned with screw pile-ups terminating at phase boundaries, again for arbitrary stress fields.
\end{abstract}

\section{Stability and Some Characteristics of Uniformly Moving Dislocations}

\author{
M. Malén
}

AB Atomenergi, Studsvik, Sweden

\begin{abstract}
The stability of uniformly moving dislocations has been studied in the case of elastic anisotropy using computer. The velocity for instability to occur for a screw dislocation in an isotropic medium is so high, $0.98 C_{t}$, with $C_{t}$ the transverse sound velocity, that it may well be unattainable. Inclusion of anisotropy gives the possibility of more reasonable instability velocities. Some data showing this for $\mathrm{Fe}$, $\mathrm{Li}$ and $\mathrm{Cr}$ are given.

The stress field around a uniformly moving dislocation has been studied. Increasing the velocity of a dislocation corresponds to some extent to a change in the anisotropy of the crystal.

In uniform motion above the lowest velocity of sound in the direction of motion one can have associated with the moving dislocation 2,4 or 6 Çerenkov waves, above the next sound velocity 4 or 6 and above the highest sound velocity 6 waves.

The formula developed earlier for the stress field around a uniformly moving planar dislocation loop can be generalized directly to three dimsions using the theorems of Indenbom and Orlov for the fields from three-dimensional sources using data for two-dimensional sources.

The generalization can also be made using the fact that the choice of cut surface is arbitrary.
\end{abstract}




\title{
Localized Vibration Modes Associated with Screw Dislocations
}

\author{
A. A. Maradudin \\ Department of Physics, University of California, Irvine, California 92664
}

The dispersion relation for the one-dimensional continuum of localized modes associated with a screw dislocation is obtained in the long wavelength limit, as a function of the wave vector parallel to the dislocation line. The result has the form $\omega^{2}(q)=s^{2} q^{2}-\omega_{0}^{2} \exp (-$ const. $/ q)$ where $s$ is the speed of sound for transverse acoustic waves, and $\omega_{0}$ is a typical Brillouin zone boundary frequency.

\section{Propagation of Glide Through Internal Boundaries}

\author{
M. J. Marcinkowski \\ Engineering Materials Group and Department of Mechanical Engineering, University of Maryland, \\ College Park, Maryland 20742
}

\begin{abstract}
It has been shown that when an internal boundary such as a grain boundary is cut by a crystal glide dislocation, a disturbance is left at the boundary. This disturbance closely resembles that about a crystal dislocation with the exception that a) there is no extra half plane associated with the dislocation and b) the Burgers vector associated with this disturbance is a variable which depends on the nature of the internal boundary. These boundary dislocations have been termed virtual dislocations.

The nature of the virtual boundary dislocations have been treated in detail for the symmetrical tilt boundary. Both homogeneous and heterogeneous type glide across these boundaries have in turn been applied to grain boundary crack formation and propagation, grain boundary rotation, preferred orientation etc.
\end{abstract}

\section{A Source of Dissipation that Produces an Internal Friction Independent of the Frequency}

\author{
Warren P. Mason
}

Department of Engineering and Applied Science, Columbia University, New York, N.Y., 10027

\footnotetext{
Many measurements of the internal friction of metals and other materials such as the earths crust show that there is a component at low frequencies which produces a value independent of the frequency. It has been shown that this component is associated with dislocation motion.

Using a model for which dislocation motion results from the motion of kinks, it is shown that such a loss can be associated with the energy dissipated when kinks cross Peierls barriers. Theoretical calculations have shown that the energy dissipated in mechanical vibrations requires a dissipative force equal to from 0.01 to 0.1 of the Peierls stress to replace the energy lost. At the low stresses used in internal friction measurements, it requires a thermal activation to cause motions of the kinks. The lag of the motion behind the applied stress produces a drag coefficient $B$ proportional to the temperature. The energy due to kink dissipation produces an internal friction to modulus change ratio $b$, equal to the ratio of the dynamic to the static kink stress. Measurements in copper indicate that this ratio is about 0.04 in agreement with calculations.
} 


\title{
Non-Planar Dissociations of Dislocations
}

\author{
S. Mendelson
}

2660 Somerset Blvd., Troy, Michigan 48084

\begin{abstract}
Non-planar dissociations of dislocations are studied in hep, fcc, bcc, diamond lattice, tetragonal and orthorhombic crystal structures. The geometric and energetic conditions are shown to be favorable for various dissociations in each crystal structure. A general equation is formulated for dissocations into partials which are glissile on various twin planes of a common zone. The Burgers vector of the twinning dislocations are expressed in terms of orthogonal unit vectors which lie in the "plane of shear" of the twin mode. The twinning dislocations are generally of the "zonal" type, chosen to be consistent with minimum shear-strain and simple atomic shuffling criteria for twinning, and applied in derivations of the twinning elements and shear-strains for various twin modes. The sign of the shear-strain determines the "stress sense" characteristics for dislocation resistance and twinning and are shown to be consistent with behavior in various hep and bcc metals. The maximum repulsive force on the twinning partials $\gamma_{m}$ is computed using anisotropic elasticity, and compared with evaluations of twin lamella energies $\gamma$. In many cases it is found that $\gamma_{m} / \gamma>1$, leading to an increase in dislocation resistance, locking, or twinning at lower temperatures. In the cases where $0<\gamma_{m} / \gamma<1$ the partialized dislocation model reduces to the “modified pseudo-Peierls-Nabarro model” for dislocation resistance.

Among various effects, the dissociations account for all twin modes in hcp metals and for the extreme difference in the flow behavior of $\mathrm{Cd}$ and $\mathrm{Zn}$ on one hand and $\mathrm{Ti}$ and $\mathrm{Zr}$ on the other. The stress dependent activation energies for motion of dissociated $60^{\circ}$ dislocations in germanium are computed and compare favorably with the data of Kabler. A "lock" for kinks on $60^{\circ}$ dislocations is discribed which can account for dragging points in the model of Celli et al.
\end{abstract}

\section{Derivation of a Continuum Theory of Dislocations on the Basis of an Estimative Analysis of Crystal Lattices}

\author{
M. Mişicu \\ Center of Mechanics of Solids, Academy of Sciences, Bucharest, Rumania \\ Starting from the non-primitive crystal lattice model, a continuum theory is derived by means of an \\ interpolation procedure which takes into account the variability of kinematic and dynamic fields. The \\ non-holonomy of the fields of displacements, energy and entropy is considered in order to elaborate the \\ analysis of dislocation and irreversible effects.
}

\section{The Elastic Field of Moving Dislocations and Disclinations}

\author{
T. Mura
}

Department of Civil Engineering and Materials Research Center, Northwestern University, Evanston, Illinois 60201

The previous work and the present stage of study on the elastic field of moving dislocations and disclinations are reviewed. Emphasis will be placed.upon the general method and approach using Green's tensor functions and Fourier integrals. The previous solutions for individual problems are reviewed as examples of this unified approach. 


\title{
Disclinations in Surfaces
}

\author{
F. R. N. Nabarro \\ Department of Physics, University of the Witwatersrand, Johannesburg, South Africa \\ The screw disclination is essentially a 2-dimensional object. Screw disclinations provide a con- \\ venient classification of star polygons. By considering the disclinations in vector fields lying on surfaces, \\ it is possible to relate Euler's theorem for polyhedra inscribed on a sphere (faces - edges + corners $=$ \\ 2) to the fixed-point theorem for continuously-varying small displacements of points on a sphere. The \\ changes in the Euler characteristic produced by the addition of holes, handles and cross caps are related \\ to the disclinations which these singularities introduce into vector fields lying on the surface. These dis- \\ clinations may be localized into the neighbourhood of their corresponding singularities.
}

\section{Eigenfrequencies in a Dislocated Crystal}

\author{
T. Ninomiya \\ Department of Physics, University of Tokyo, Tokyo, Japan
}

\begin{abstract}
Dynamical theories of dislocation vibration and interactions with phonons are surveyed. Eigenfrequencies of lattice vibration in a crystal containing a straight dislocation vibration (wave number $\kappa$ ) in each of the intervals of the normal mode frequencies of $k_{z}=\kappa$ in a perfect lattice. It is also found that there is a band of localized dislocation vibration below the phonon band. The mean squared amplitude of the dislocation vibration is determined by the localized mode for an edge dislocation and by the resonance modes for a screw dislocation. Phonon scattering by the fluttering mechanism is next treated by using the above results and the condition of resonance scattering is given. Finally, the effect of the Peierls potential and the vibration of a dislocation dipole are discussed. In the Appendices the problem of quantization of dislocation vibration and the extension of the above theories to a case of translational motion is briefly described.
\end{abstract}

\section{Electronic Effects Associated with Stacking Faults in Normal Metals}

\author{
C. Nourtier
}

Physique du Solide, Faculté des Sciences, 91 Orsay, France

and

\section{G. Saada}

\section{Département de Physique, CUSLA, B.P. 36, 59-Lille Distribution, France}

The authors use a simple pair interaction model to make the following calculations. 1) Stacking fault energy of simple metals. 2) Interaction energy between a stacking fault and an impurity atom or a vacancy, and application to the study of segregation effects. 3) Interaction energy of parallel stacking faults.

Numerical results are given. Consequences of the long range interaction between defects are discussed. 


\title{
Dislocation Pair Interaction in a Finite Body
}

\author{
R. O. Scattergood and U. F. Kocks
}

\section{Metallurgy Division, Argonne National Laboratory, Argonne, Illinois 60439}

The elastic interaction between dislocations in a finite body shows important features not found in an infinite body. For example, two like dislocations parallel to the axis of a cylinder repel each other only when they are close, but attract each other when their distance is comparable to the cylinder radius. Contours of the pair interaction energy are given for both screw and edge dislocations in a circular cylinder.

These new features appear not only when free surface conditions are demanded on the entire body, but even when approximate stress fields are used that demand some surface tractions, so long as no torques are transmitted through the body.

The relevant torques (bending moments) associated with an edge dislocation in a circular cylinder, which vanish when the mantle is traction free, may be made to vanish in general by a simple modification of the stress field of the edge dislocation.

\section{Dislocation Radiation}

\author{
R. O. Schwenker and A. V. Granato \\ Department of Physics, University of Illinois, Urbana, Illinois 61801
}

\begin{abstract}
Thin walls of mobile dislocations have been produced. These can be excited to emit macroscopic plane sound waves. Calculations have been made to predict the properties of the reradiated waves on the basis of a vibrating string model which neglects dislocation interactions. Measurements of the relative modulus change $\Delta G / G$ and the decrement $\Delta$ (real and imaginary part of the response) as a function of frequency permit a check of the Kramers-Kronig dispersion relations. In addition, measurements of the amplitude of the reradiated wave provide another check since the amplitude is proportional to $\left[(\Delta G / G)^{2}+(\Delta / \pi)^{2}\right]^{1 / 2}$.
\end{abstract}

\section{Dragging Forces on Moving Defects by Strain-Field Phonon Scattering}

\author{
A. Seeger and H. Engelke \\ Max-Planck-Institut für Metallforschung, Institut für Physik, Stuttgart, and Institut für Theoretische \\ und Angewandte Physik der Universität, Stuttgart, Germany

\begin{abstract}
An expression for the dragging force on a uniformly moving defect by scattering of phonons at its strain-field has been derived using nonlinear elasticity theory. The quantization procedures and the formulation of the master equation for the phonon distribution follow the techniques developed in the theory of heat conductivity. Numerical calculations have been performed for kinks in screw dislocations in copper. A comparison with numerical results obtained in the theory of heat conductivity shows quite good agreement. The formalism developed should prove useful also for calculations of the electron drag on dislocations in metals.
\end{abstract}




\title{
Screw Dislocations inn Inhomogeneous Solids
}

\author{
G. P. Sendeckyi
}

School of Engineering, Case Western Reserve University, Cleveland, Ohio 44106

The problem of screw dislocations interacting with free surfaces and inhomogeneities is reconsidered and a general method is presented for solving a large class of screw dislocation problems. The method is based on knowledge of certain general solutions in the theory of antiplane deformation of elastic solids. It can be shown that all the known screw dislocation solutions for solids undergoing antiplane deformation can be found by using thiss approach.

As an illustration of the method, three new solutions for screw dislocations near inhomogeneities are given. These are the screw dislocation near 1) an elastic elliptic cylindrical inclusion, 2) two circular cylindrical inclusions, and 3) a curvilinear cavity or rigid inclusion. The interaction energy between the dislocation and the inhomogeneities is also computed.

\section{A Geometric Foundation for the Kinematics of the Imperfect Continuum}

\author{
John A. Simmons
}

Metallurgy Division, National Bureau of Standards, Washington, D.C. 20234

This investigation is concerned with the foundations of the geometry of a continuum approximation to the deformation of an imperfect crystalline solid.

A method of formulating the infinitesimal deformation of a system of particles based on an averaging process is developed. This formulation is based on the construction of a "polyhedral mesh" valid for any system of particles whether or not they lie in a lattice configuration. When the particles lie in a perfect lattice configuration, the mesh formalism is shown to reproduce the kinematics of a classical elastic continuum. If the lattice configuration is not perfect two alternate kinematic descriptions in terms of space-time affine connections are derived by the averaging process. The simpler produces a curvature free formalism corresponding to the distant parallelism formulation of Bilby et al. This formulation is seen to be appropriate when the local elastic energy density is essentially determined by the averaged local lattice distortion such as might be expected in non-polygonized single crystals. The second kinematic description produces an averaged local lattice distortion together with a measure of dispersion of this distortion. This gives rise to a dynamic affine connection with curvature. This type of description is seen to be more appropriate to polycrystalline and polygonized single crystalline materials.

Using these results, the concept of infinitesimal plastic transformation is formulated and it is shown that by assuming the initial state of the material to be described by a spatial affine connection, the entire dynamic description of the mateiral deformation is then given by a four dimensional space-time affine connection whose invariants together with the integrated strain define the state of the material. Equations of continuity for plastic as well as for ordinary elastic deformation are derived. The exterior calculus of E. Cartan is utilized in this section to simplify the computations. 


\title{
Internal Stress and the Incompatibility Problem in Infinite Anisotropic Elasticity
}

\author{
John A. Simmons \\ Metallurgy Division, National Bureau of Standards, Washington, D.C. 20234 \\ and
}

R. Bullough

Theoretical Physics Division, A.E.R.E., Harwell, Didcot, Berkshire, England

\begin{abstract}
Using the language of projection operators, the elastic distortion field of a linear infinite anisotropic body is decomposed into its internal and external components. The kernel of the external projection operator is identified as the elastic field due to force dipoles while that of the internal field corresponds to internal distortion fields due to displacement dipoles.

By integration of the projection operator for the internal distortion field, an alternative description for internal distortion fields in terms of dislocations is given. The Mura-Willis formula as well as the distortion field due to a rational dislocation element (in the sense of Eshelby and Laub) for an anisotropic body are then obtained as integrals of the basic displacement dipole kernel for internal distortions.

Further integration of the displacement dipole kernel provides a description of the internal distortion field due to a rational incompatibility element. The general formula for the stress function due to an incompatibility distribution in a general infinite anisotorpic body is then given and shown to reduce to the formulation given by Kröner for isotropic bodies.

Finally, explicit methods to compute kernels for internal distortions due to incompatibilites are given and discussed.
\end{abstract}

\section{The Behavior or an Elastic Solid Containing Distributions of Free and Fixed Dislocations}

\author{
E. Smith \\ Department of Metallurgy, University of Manchester, England
}

\begin{abstract}
There are many situations in metal physics where the stresses acting on fixed dislocations have an important bearing on a physical phenomenon, and the paper derives a general expression relating these stresses when fixed edge dislocations are contained within an infinite elastic solid in which there are also free edge dislocations that occupy equilibrium positions.

Special cases are considered in detail, particular attention being given to the situation where all the dislocations are of the same type, the free ones having identical Burgers vectors $b$ while there are two fixed dislocations with Burgers vectors $\mathrm{pb}$ and $\mathrm{gb}$; all the dislocations lie in the same plane within an infinite solid. This is the most general model for which the stresses on each dislocation and also the equilibrium positions of the free dislocations may be determined analytically. It is indicated how the model degenerates into all the others that have been discussed analytically in terms of classic polynomial functions.

The results are briefly discussed in relation to the problem of cleavage crack nucleation in crystalline solids.
\end{abstract}




\title{
The Elastic Generalized Cosserat Continuum with Incompatible Strains
}

\author{
Rastko Stojanović
}

\author{
Department of Mechanics, University of Belgrade, Yougoslavia
}

\begin{abstract}
Considered is a continuum with deformable directors. Dislocations are regarded as sources of incompatibilities and internal strains. Established are the relations between the dislocation density tensor and the gradients of directors. The conservation law for dislocations (i.e. the law that the dislocation lines cannot end inside a body) appears as the integrability condition for the dislocation density - gradients of directors relation. In a linearly connected space $L_{3}$ in which the directors of a dislocated medium represent fields of absolutely parallel vectors the equality of the dislocation density tensor with the torsion tensor of the space $L_{3}$ follows as an immediate consequence. It is also proved that the geometry of $L_{3}$ is equivalent to the geometry of the linearly connected space $L_{3}$ which is defined in terms of the distorsions, introduced in the theories based on the non-oriented continuum models. From the principle of virtual work are derived the general (non-linear) relations for stress and hyperstress. The stress tensor is not symmetric. In the appendix are presented the modified divergence theorem and the expressions for the variations of the gradients of directors in the absence of a displacement field, i.e., in the case when the compatibility conditions for the strain tensor are not satisfied.
\end{abstract}

\section{The Broadening of Resonance Lines by Dislocations}

\author{
A. M. Stoneham \\ Theoretical Physics Division, A.E.R.E., Harwell, Didcot, England and Coordinated Science Laboratory, \\ University of Illinois, Urbana, Illinois 61801

\begin{abstract}
Dislocations and other defects in solids broaden sharp resonance lines in solids, such as spin resonance and optical zero phonon lines. The broadening is a result of the random strain fields produced, and the shape of the resonance line is a measure of the distribution of internal strains. Measurement of line shapes can give information which is different from that given by the more usual mechanical and transport studies of dislocations. We calculate the shapes of the resonance lines in terms of the properties of the individual defects which cause broadening, their density and their statistical distribution. The theory is applied to straight dislocations, infinitesimal loops, dislocation dipoles and to point defects.
\end{abstract}

\section{Effect of Zero-Point Motion on Peierls Stress}

\author{
H. Suzuki
}

Department of Physics, Faculty of Science, University of Tokyo, Bunkyo-ku, Tokyo, Japan

Calculations of the Peierls stress hitherto made are criticized and the following conclusions are obtained. The significant difference in Peierls stress between different materials arises mainly from the difference in crystal structures. The Peierls stress is necessarily high in a rectangular lattice where atoms just above and below the slip plane face each other, while in the lattices where the atoms face alternately along the slip plane it is of the order of one percent of that in the rectangular lattice. The Peierls stress in the body-centered cubic crystal is, however, rather high for a screw dislocation owing to the screw structure of this crystal with the axes parallel to [111] direction. The calculated Peierls 
stresses are several times of those expected from experiments. The zero-point motion decreases the calculated Peierls stress through two mechanisms. The one is the difference in frequency spectrum of a dislocation line at the bottom of the potential valley and at the top of the potential hill. The other is due to the change in spring constants of atom pairs around the dislocation through anharmonicity.

\title{
A Dynamic Theory of Dislocations and its Applications to the Theory of the Elastic-Plastic Continuum
}

\author{
C. Teodosiu* \\ Max-Planck-Institut für Metallforschung, Institut für Physik, Stuttgart, and Institut für Theoretische
und Angewandte Physik der Universität, Stuttgart, Germany

\begin{abstract}
The starting point of this study is a paper by Kröner (J. Math. Phys., 42, p. 27, 1963), who proposed the replacement of the loading history variables in the yield criterion and in the work-hardening equation by state variables, such as the dislocation density and the density of dislocation loops.

The first part of the work summarizes the formulation recently given by the author for the dynamic non-linear theory of dislocations in anisotropic media. The relations connecting the continuum kinematic quantities to the discrete dislocation arrangement and to the dislocation velocities are emphasized.

To construct a theory of the elasto-plasticity, the system of kinematic equations is completed by postulating a yield condition and a work-hardening equation, which contain as independent variables the stress, the dislocation density, the density of dislocation loops, and the temperature.

Making constitutive assumptions for the free energy, the specific entropy, and the heat conduction vector, and using the principle of material indifference and the thermophysical restrictions, a definite theory of the elastic-plastic continuum is obtained.
\end{abstract}

\section{Non-Linear Dynamic Problems for Anisotropic Elastic Bodies in the Continuum Theory of Dislocations}

\author{
C. Teodosiv* and A. Seeger
}

\author{
Max-Planck-Institut für Metallforschung, Institut für Physik, Stuttgart, and Institut für Theoretische \\ und Angewandte Physik der Universität, Stuttgart, Germany
}

\begin{abstract}
The non-linear continuum theory of dislocations has been used to solve a number of problems for isotropic materials, including the determination of stresses produced by dislocations, the scattering of elastic waves by straight dislocations and kinks, and the small-angle scattering of X-rays by dislocation lines and rings. For the application of these results it is necessary to know the elastic constants of third or higher order entering the constitutive equations. Third-order elastic constants have recently become available for a large class of single crystals with an accuracy that exceeds considerably the earlier polycrystalline data. It appears therefore desirable to develop a formulation of the theory that may be applied to anisotropic materials.

The present work aims at developing approximate methods for solving non-linear problems such as the determination of the strains produced by stationary or uniformly moving dislocations in anisotropic media and of their effects on a superimposed infinitesimal motion.

The non-linear problems are solved by reduction to a series of linear problems using expansions in terms of small parameters. Three such parameters appear naturally: the magnitude of the distortion produced by dislocations, the magnitude of the deformation gradient of the superimposed motion, and the ratio between the dislocation and sound velocities. To obtain definite approximation algorithms, hypotheses concerning the relative order of magnitude of these three parameters are introduced.
\end{abstract}

*Permanent address: Center of Mechanics of Solids, Str. Constantin Mille 15, Bucharest, Romania 


\title{
Kinks, Vacancies, and Screw Dislocations
}

\author{
Robb M. Thomson
}

Department of Materials Science, State University of New York at Stony Brook, Stony Brook, New York 11790

\begin{abstract}
A vacancy on a nonsplit pure screw dislocation can dissociate into a set of kinks. This dissociation is demonstrated geometrically for the $\mathrm{NaCl}$ lattice, showing that no geometrical constraints are violated by the dissociation. The kinks thus generated also splinter and spread the charge of the vacancy along the line. The effective vacancy association energy on the line is thus much higher than has been supposed hitherto, and is partly due to the delocalization of the charge singularity of the point defect and partly due to the delocalization of elastic singularity. When the Peierls energy is low, the vacancy will always dissociate, while if it is high, the dissociation will occur only when the total kink energy is less than the vacancy energy. Vacancy contributions to both climb and pipe diffusion are discussed in terms of the kink dissociation process. Results are that interstitial pipe diffusion is entirely summetric to vacancy pipe diffusion, no motion energy is needed, and the formation energy for diffusion is related to the Peierls energy.
\end{abstract}

\section{Theory of Surface States on Stacking Faults}

\author{
Robb M. Thomson
}

Department of Materials Science, State University of New York at Stony Brook, Stony Brook, New York 11790

The Slater-Koster method in the Bloch representation is applied to a model for a stacking fault in an idealized crystal leading to a type of Shockley surface state. A series expansion is obtained for the energy which is worked out for the simplified case of a constant matrix element, with the result that for a tight binding nonoverlapping band, there are surface waves which split off from both the top and bottom of the band. Contrary to Shockley's results for external surfaces, and to the Slater-Koster theory of the localized impurity, localized states occur on the stacking fault for a single nonoverlapping band and for arbitrary potential values. A constant matrix approximation is worked out for the case of the simple metals.

\section{The Elastic Interaction Between Grain Boundaries and Screw Dislocation Pile-Ups}

\author{
M. O. Tucker \\ Department of Physics, University of Surrey, Guildford, Surrey, England
}

\begin{abstract}
The configuration of an array of parallel infinitive straight screw dislocations, in equilibrium under a constant applied stress, and pile-up on a plane inclined to a grain boundary at an arbitrary angle is considered. The model used for the grain boundary is the plane interface between two elastically anisotropic half-spaces welded together. Using this approximation of a continuous distribution of infintesimal dislocations the integral equation expressing the equilibrium conditions is solved using a Wiener-Hopf technique and approximate expressions are presented for the stresses near to the tip of the array when the dislocations are parallel to orthotropic symmetry axes in each half-crystal.
\end{abstract}




\title{
On the Thermodynamics of Inhomogeneous Bodies
}

\author{
C.-C. Wang
}

\author{
Department of Mathematical Sciences, Rice University, Houston, Texas 77001
}

\begin{abstract}
This paper concerns the thermodynamics of inhomogeneous elastic bodies. I adopt the constitutive equations for thermoelastic materials given by Coleman \& Noll (1963) and develop a theory for bodies made up of such materials. The general scheme of this theory is based on my paper on Generalized Simple Bodies (1969). The main result is the explicit field equations for the deformation and the temperature on a thermoelastic body.
\end{abstract}

\section{Subsonic, Supersonic, and Transonic Dislocations Moving on an Interface Separating Two Media of Differing Elastic Properties}

\author{
J. Weertman, H. M. Berg, J. E. Bloom, H. Ishii, R. H. Marion, D. E. Pease, D. T. Spreng, \\ and J. B. Vander Sande*
}

Materials Science Department and Materials Research Center, Northwestern University, Evanston, Illinois 60201

This paper examines the problem of a dislocation moving on an interface separating two isotropic elastic media that have differing elastic constants and densities. This problem has application to the phenomenon of diffusionless transformations. Solutions are found for moving screw dislocations, gliding edge dislocations, and climbing edge dislocations. It is assumed that the dislocation velocity lies in either the subsonic, the transonic, or the supersonic velocity region. We have generalized the analysis that was used in a study of the elastic displacements and stress field of subsonic, transonic, and supersonic dislocations moving in an ordinary elastic medium. The results given in the present paper are formally identical to those obtained in that simpler analysis.

\section{Thermal Energy Trapping by Moving Dislocations}

\author{
J. H. Weiner \\ Division of Engineering, Brown University, Providence, R. I. 02912
}

\begin{abstract}
The steady motion of a dislocation along a piece-wise harmonic Frenkel-Kontorowa model is considered. For suitable model parameters there is one localized mode associated with either the stable or unstable dislocation configuration and the remaining modes are non-localized or extended. Because of the piece-wise harmonic character of the model, the set of normal modes of the system changes at discrete instants of time, referred to as transition times, as the dislocation moves along the chain. In particular, the localized modes must move along with the dislocation position and we refer to the localized mode momentum and energy as the dislocation momentum and energy respectively. The particle momentum and energy due to the sum of the extended modes is termed thermal.

At the transition times, it is necessary to expand atomic velocities in terms of the new set of modes appropriate to the forthcoming state of the crystal. It is found that a coordination effect exists between the transition times and the thermal motion such that on the average over many transitions, thermal momentum in the direction of the dislocation motion is transferred to the dislocation momentum.
\end{abstract}

${ }^{*}$ Instructor and students of Advanced Dislocation Class at Northwestern University. 


\title{
The Mean Square Stresses $\left\langle\sigma^{2}\right\rangle$ for a Completely Random and a Restrictedly Random Distribution of Dislocations in a Cylindrical Body
}

\author{
M. Wilkens
}

\author{
Max-Planck-Institut für Metallforschung, Institut für Physik, Stuttgart, Germany
}

The paper gives a full proof of the author's earlier statement according to which the elastic interaction energy of completely randomly distributed dislocations vanishes. As a consequence the outer cut-off radius in the logarithmic factor of the elastic energy coincides with the external cylinder radius rather than with the mean dislocation spacing. The proof takes into account the elastomechanical boundary conditions which were neglected in the earlier paper. Further it is shown that for a restrictedly random distribution the outer cut-off radius is equivalent to the radius of the areas small compared with the cylinder cross-section, each of which contains the same number of dislocations randomly distributed over the corresponding area. In the special case that each area contains only one dislocation with arbitrary inside the outer cut-off radius degenerates to the mean dislocation spacing.

\section{Theoretical Aspects of Kinematical X-Ray Diffraction Profiles from Crystals Containing Dislocation Distributions}

\author{
M. Wilkens
}

\begin{abstract}
Max-Planck-Institut für Metallforschung, Institut für Physik, Stuttgart, Germany
\end{abstract}

\begin{abstract}
The paper deals with a theoretical study of the Fourier transorm of the X-ray diffraction line profiles from crystals containing dislocation distributions. The theory is based mainly on a calculation of spatial averages of powers of the strains $\epsilon_{n}$, where $\epsilon_{n}$ means the differential strain perpendicular to the reflecting planes averaged over a certain length $n$ ( $n=$ variable of the Fourier transform). The second power average $\left\langle\epsilon_{n}{ }^{2}\right\rangle$ is calculated in a closed form for a restrictedly random dislocation distribution. However, the result is approximately valid also for other distributions. The contributions of the averages of higher powers of $\epsilon_{n}$ are taken into account in an approximate manner. The expression derived for the Fourier transform depends on the two parameters $\rho$ and $R_{e}$, where $\rho$ is the dislocation density. $R_{e}$ is equivalent to the effective outer cut-off radius which appears for instance in the logarithmic factor of the elastically stored energy. The result which is applicable for $R_{e} \geq \rho^{-1 / 2}$ is discussed in comparison with the theory of Krivoglaz and Ryaboshapka, in which a completely random dislocation distribution is assumed, and with the theory of diffraction from distorted crystals mainly developed by Warren and Averbach.
\end{abstract}

\section{Structural and Elastic Properties of Zonal Twin Dislocations in Anisotropic Crystals}

\author{
M. H. Yoo and B. T. M. Loh
}

Metals and Ceramics Division, Oak Ridge National Laboratory, Oak Ridge, Tennessee 37830

A descriptive definition of zonal twin dislocations for compound twin systems is given based on the well established rational crystallographic elements. Geometric characteristics of zonal twin dislocations in double lattice structures are thoroughly discussed. Equilibrium shapes of an incoherent twin boundary have been analyzed by using the anisotropic elastic properties of edge dislocations. Short-ranged structural properties of zonal twin dislocations are discussed based on a Peierls-Nabarro model. It is found that the "anisotropic parameter," $K_{e} S_{66}$, correctly predicts the active mode of crystallographically non-equivalent conjugate twin systems. 